\title{
A Bayesian View on the Hilbert Transform and the Kramers- Kronig Transform of Electrochemical Impedance Data: \\ Probabilistic Estimates and Quality Scores
}

Jiapeng Liu ${ }^{\mathrm{a}, 1}$, Ting Hei Wan ${ }^{\mathrm{a}, 1}$, Francesco Ciucci ${ }^{\mathrm{a}, \mathrm{b}, \uparrow}$

a Department of Mechanical and Aerospace Engineering, The Hong Kong University of Science and Technology, Hong Kong, China

${ }^{\mathrm{b}}$ Department of Chemical and Biological Engineering, The Hong Kong University of Science and Technology, Hong Kong, China

${ }^{\dagger}$ Corresponding author: francesco.ciucci@ust.hk

${ }^{1}$ These authors contributed equally to this work

Keywords: Electrochemical Impedance Spectroscopy, Distribution of Relaxation Times, Ridge/Tikhonov Regularization, Bayesian Statistics, Lithium-ion Batteries 


\section{Abstract}

Electrochemical impedance spectroscopy (EIS) is one of the most widely used experimental tools in electrochemistry and has applications ranging from energy storage and power generation to medicine. Considering the broad applicability of the EIS technique, it is critical to validate the EIS data against the Hilbert transform (HT) or, equivalently, the KramersKronig relations. These mathematical relations allow one to assess the self-consistency of obtained spectra. However, the use of validation tests is still uncommon. In the present article, we aim at bridging this gap by reformulating the HT under a Bayesian framework. In particular, we developed the Bayesian Hilbert transform (BHT) method. We also proposed several scores that can provide a quick metric for the evaluation of the EIS data quality.

Keywords: Electrochemical Impedance Spectroscopy, Hilbert Transform, Kramers-Kronig Relations, Bayesian Methods, Quality scores 


\section{Introduction}

Electrochemical impedance spectroscopy (EIS) is one of the most important and versatile techniques of electrochemistry.[1] EIS has been used widely in the fields of energy storage, [2, 3] solid-state ionics, [4, 5] fuel cells, [6, 7] electrolyzers,[8] solar cells, $[9,10]$ porous media,[11] sensors,[12] biology,[13] virological diagnostics,[14] and medicine.[15, 16] The EIS technique is particularly appreciated because it can be carried out for frequencies spanning several orders of magnitude, typically from $1 \mathrm{mHz}$ to $10 \mathrm{MHz}$. Obtaining information across such a broad range of timescales allows one to gain insights from many disparate physicochemical phenomena.[17]

The impedance measured by the EIS technique is a transfer function, and, as such, it needs to satisfy linearity, time-invariance, and causality.[1, 18] Compliance with these properties can be evaluated experimentally, for example, by varying the EIS measurement settings systematically[19] or by broadband excitation.[20] However, these testing procedures are not necessarily possible or may take an unnecessarily long time. Alternatively, one could assess whether the measured EIS spectrum satisfies the criteria mentioned above using the KramersKronig (KK) relations.[21, 22] Such relations, which can be obtained by manipulating the Hilbert transform (HT) of suitable even and odd functions, link the real and imaginary parts of the impedance to one another through integrals over frequencies from 0 to $\infty$. While the exact implementation of KK relations needs impedance data for all possible frequencies, the EIS spectra, in reality, are only discretely sampled over a finite interval of frequencies. Methods, including direct integration and regression of generalized circuit models, [18, 23-29] have been developed to overcome this limitation. Consequently, the KK relations have been available as 
a tool to assess the validity of the EIS spectra. For an excellent review of the use of KK relations applied EIS, the reader is referred to the textbook of Orazem and Tribollet[1] and the monograph by Lasia.[30] We should remark that the EIS spectra are rarely benchmarked against the KK relations and therefore tested for their validity.

In this article, we aim at overcoming this significant gap by taking a slightly different starting point compared to the ones of the KK relations. We are going to focus on the HT, which, in the context of the transfer functions and therefore, the EIS, is equivalent to the KK relations.[31] The HT is used in many applications, including fluid mechanics,[32] aerodynamics,[33] optics,[34] and geophysics.[35] Relative to the KK relations, the HT has far richer mathematical literature, with the availability of fast HT methods[36, 37] and many theoretical results.[31, 38, 39] In the context of analyzing the consistency of EIS data, we will set up a new framework, which is named as Bayesian Hilbert transform (BHT). As a first step, the BHT approach uses a linear approximation of the impedance, $Z(\omega)$, with respect to a given basis. In other words, we will write $Z(\omega)=\sum_{n} x_{n} \psi_{n}(\omega)$, where the $\psi_{n}(\omega)$ 's are transfer functions and $x_{n}$ 's are random variables (RVs) endowed with a certain probability distribution function (pdf) that will need to be determined. Here, we will use the $\psi_{n}(\omega)$ 's originating from the distribution of relaxation times (DRT) and take the $x_{n}$ 's to be normally distributed. After having regressed the $x_{n}$ 's, we will be able to compute $Z_{\mathrm{H}}(\omega)$, i.e., the HT of $Z(\omega)$ using a simple matrix multiplication. An important point to note is that both $Z(\omega)$ and $Z_{\mathrm{H}}(\omega)$ will be understood as normally distributed RVs. Doing so will allow us to use analytical formulas and define a number of scores that quantify consistency of the real and imaginary parts of the regressed $Z(\omega)$ against experimental data and with themselves. 
The scientific contributions of the paper are twofold. First, we reframed the HT of the EIS data in a general Bayesian context, where we leverage analytical matrix expressions. This allowed us to determine the hyperparameters used in the regression by maximizing the evidence rather than by an ad hoc guess. Second, we proposed new ways to score the compliance of $Z(\omega)$ with the HT. These scores are based on residual plots, mean discrepancies, and "distances" between the pdfs of $Z(\omega)$ and $Z_{\mathrm{H}}(\omega)$. We have also shared our code and included some of the developed metrics in DRTtools.[40] We expect that doing so will promote the inclusion of the HT or KK test of EIS data in existing software packages. Lastly, we must point out that, despite their importance, the KK relations are not widely used in the electrochemistry field. This is perhaps due to the difficulty in understanding the theory and the availability of modern software. We hope that our contribution will be instrumental in overcoming these two challenges.

\section{Theory}

\subsection{Hilbert Transform}

In this section, we define the HT and recall how to link HT to KK relations. For an authoritative review of the topic, interested readers are invited to consult King's two-volume book.[31] The HT on the real line is defined as the operator that transforms a function $f(\omega)$ into another function $H f(x)$ via the following integral:

$$
H f(\omega)=\frac{1}{\pi} P \int_{-\infty}^{\infty} \frac{f(\widehat{\omega})}{\omega-\widehat{\omega}} d \widehat{\omega}
$$

where the symbol $P \int(\cdot) d \widehat{\omega}$ denotes the Cauchy principal value, which is formally defined as 


$$
P \int_{-\infty}^{\infty} \frac{f(\widehat{\omega})}{\omega-\widehat{\omega}} d \widehat{\omega}=\lim _{\varepsilon \downarrow 0} \int_{|\omega-\widehat{\omega}|>\varepsilon} \frac{f(\widehat{\omega})}{\omega-\widehat{\omega}} d \widehat{\omega}
$$

Other variants of the HT exist, including the HT on the circle, the finite HT, the multidimensional HT, and the discrete HT.[31] In this article, we will only use (1).

Let us recall a few fundamental properties of the HT applied to the EIS. If $f(z)$ is an EIS transfer function with $f(z) \rightarrow 0$ as $z \rightarrow \infty$, then

$$
\begin{gathered}
f_{\mathrm{re}}(\omega)=-H f_{\mathrm{im}}(\omega) \\
f_{\mathrm{im}}(\omega)=H f_{\mathrm{re}}(\omega)
\end{gathered}
$$

where $f_{\mathrm{re}}(\omega)=\operatorname{Re}(f(\omega))$ and $f_{\mathrm{im}}(\omega)=\operatorname{Im}(f(\omega))$ are the real and imaginary parts of $f(\omega)$, respectively. We also note that, in the context of EIS, $f_{\text {re }}(\omega)$ and $f_{\mathrm{im}}(\omega)$ are even and odd, respectively.[1] Enforcing one of the two equations in (3) implies that the other is satisfied.[31] Therefore, meeting (3) is equivalent to fulfilling the KK relations, which are the even and odd HTs.[1, 31]

Lastly, we wish to stress on a notational point. When we have a function $g(\omega, \boldsymbol{\theta})$ depending on multiple variables and want to take the HT of $g(\omega, \boldsymbol{\theta})$ with respect to $\omega$, we will explicitly indicate the variable used in the transformation by adding a subscript under the $H$ symbol. For example, for $g(\omega, \boldsymbol{\theta})$, we will designate with $H_{\omega} g(\omega, \boldsymbol{\theta})$ the following:

$$
H_{\omega} g(\omega, \boldsymbol{\theta})=\frac{1}{\pi} P \int_{-\infty}^{\infty} \frac{g(\widehat{\omega}, \boldsymbol{\theta})}{\omega-\widehat{\omega}} d \widehat{\omega}
$$




\subsection{The Bayesian Hilbert Transform of Impedance Data}

In this section, we describe how the BHT of EIS data is formulated. The BHT methodology is schematically illustrated in Figure 1. The cornerstone of the method is to approximate the impedance using a finite expansion over a latent vector $\boldsymbol{x}$. Then, from $\boldsymbol{x}$, we compute the HT by matrix multiplication. Throughout, we will use Bayesian statistics. That is, we will attach a Gaussian probability distribution to the regressed impedance and the predicted HT by assuming that the latent $\boldsymbol{x}$ follows some prior pdf. Most importantly, we provide a set of scores that gauge how experimental, regressed, and HT EIS spectra compare to one another.

\subsubsection{Approximation}

As outlined in the introduction, we will leverage an expansion of the impedance on some basis set $\{\psi(\omega, \lambda)\}$, where the $\psi(\omega, \lambda)$ 's are complex-valued transfer functions dependent on a parameter $\lambda$ such that $\lim _{\omega \rightarrow \infty} \psi(\omega, \lambda)=0$. Explicitly, we will model the impedance to be

$$
Z(\omega)=i \omega L_{0}+R_{\infty}+\int_{\mathbb{R}} \psi(\omega, \lambda) \gamma(\lambda) d \lambda
$$

where $\gamma(\lambda)$ is some other latent function to be determined. Here, we will take $\psi(\omega, \tau)=\frac{1}{1+i \omega \tau}$ and $\lambda=\log \tau$ to obtain a DRT-like approximation of the following type: [41]

$$
Z(\omega)=i \omega L_{0}+R_{\infty}+\int_{-\infty}^{\infty} \psi(\omega, \tau) \gamma(\log \tau) d \log \tau
$$

If the term $i \omega L_{0}$ is discarded, the HTs of the real or imaginary parts of the impedance can be obtained using $H_{\omega} \psi(\omega, \tau)=i \psi(\omega, \tau)$ as 


$$
\begin{aligned}
H Z(\omega) & =\int_{-\infty}^{\infty} H_{\omega}(\psi(\omega, \tau)) \gamma(\log \tau) d \log \tau \\
& =i \int_{-\infty}^{\infty} \psi(\omega, \tau) \gamma(\log \tau) d \log \tau
\end{aligned}
$$

Explicitly, if we define $\psi_{\mathrm{re}}(\omega, \tau)=\operatorname{Re}(\psi(\omega, \tau))=\frac{1}{1+(\omega \tau)^{2}}$ and $\psi_{\mathrm{im}}(\omega, \tau)=\operatorname{Im}(\psi(\omega, \tau))=$ $-\frac{\omega \tau}{1+(\omega \tau)^{2}}$, we can write that

$$
\begin{aligned}
Z_{\mathrm{re}}(\omega) & =-H Z_{\mathrm{im}}(\omega) \\
& =\int_{-\infty}^{\infty} \psi_{\mathrm{re}}(\omega, \tau) \gamma(\log \tau) d \log \tau \\
Z_{\mathrm{im}}(\omega) & =H Z_{\mathrm{re}}(\omega) \\
& =\int_{-\infty}^{\infty} \psi_{\mathrm{im}}(\omega, \tau) \gamma(\log \tau) d \log \tau
\end{aligned}
$$

Therefore, if we can estimate $\gamma(\log \tau)$ from $Z_{\text {re }}(\omega)$ or $Z_{\text {im }}(\omega)$, then we will be able to obtain $Z_{\mathrm{im}}(\omega)$ or $Z_{\mathrm{re}}(\omega)$, respectively, thanks to (8). We must stress that, in the context of this article, we will not aim to assign any physical meaning to the (latent) function $\gamma(\log \tau)$. Also, if we choose $\gamma(\log \tau)=\sum_{n=1}^{N} R_{n} \tau \delta\left(\tau-\tau_{n}\right)$, where $R_{n}$ is some parameter and $\delta\left(\tau-\tau_{n}\right)$ is a Dirac distribution centered at $\tau_{n}$, we retrieve the Voigt expansion used elsewhere in the context of the KK tests.[23, 25, 29]

To approximate $\gamma(\log \tau)$ numerically, we expand it over a finite set of functions $\mathcal{B}=$ $\left\{\phi_{1}(\log \tau), \phi_{2}(\log \tau), \ldots, \phi_{N}(\log \tau)\right\}$ as

$$
\gamma(\log \tau)=\sum_{n=1}^{N} \gamma_{n} \phi_{n}(\log \tau)
$$


where the $\gamma_{n}$ 's are scalars. By plugging (9) into (6), we can write the following two vector equations

$$
\begin{aligned}
& \boldsymbol{Z}_{\mathrm{re}}=R_{\infty} \mathbf{1}+\boldsymbol{A}_{\gamma, \mathrm{re}} \boldsymbol{\gamma} \\
& \boldsymbol{Z}_{\mathrm{im}}=L_{0} \boldsymbol{\omega}+\boldsymbol{A}_{\gamma, \mathrm{im}} \boldsymbol{\gamma}
\end{aligned}
$$

where $\boldsymbol{\gamma}=\left(\gamma_{1}, \gamma_{2}, \ldots, \gamma_{N}\right)^{\top} \in \mathbb{R}^{N}$ and the $\boldsymbol{Z}_{\mathrm{re}}, \boldsymbol{Z}_{\mathrm{im}}, \mathbf{1}, \boldsymbol{\omega} \in \mathbb{R}^{M}$ are vectors such that, for $1 \leq$ $m \leq M,\left(\boldsymbol{Z}_{\mathrm{re}}\right)_{m}=Z_{\mathrm{re}}\left(f_{m}\right),\left(\boldsymbol{Z}_{\mathrm{im}}\right)_{m}=Z_{\mathrm{im}}\left(f_{m}\right),(\mathbf{1})_{m}=1$, and $(\boldsymbol{\omega})_{m}=\omega_{m}$. Explicitly, the entries of the matrices $\boldsymbol{A}_{\gamma, \mathrm{re}}, \boldsymbol{A}_{\gamma, \mathrm{im}} \in \mathbb{R}^{M \times N}$ are defined as

$$
\begin{aligned}
& \left(\boldsymbol{A}_{\gamma, \mathrm{re}}\right)_{m n}=\int_{-\infty}^{\infty} \psi_{\mathrm{re}}(\omega, \tau) \phi_{n}(\log \tau) d \log \tau \\
& \left(\boldsymbol{A}_{\gamma, \mathrm{im}}\right)_{m n}=\int_{-\infty}^{\infty} \psi_{\mathrm{im}}(\omega, \tau) \phi_{n}(\log \tau) d \log \tau
\end{aligned}
$$

We note that these two matrices may be obtained with any of the methods described elsewhere.[40, 42]

For notational convenience, we will define the following two matrices:

$$
\begin{gathered}
\boldsymbol{A}_{\mathrm{re}}=\left(\mathbf{1}, \boldsymbol{A}_{\gamma, \mathrm{re}}\right) \\
\boldsymbol{A}_{\mathrm{im}}=\left(\boldsymbol{\omega}, \boldsymbol{A}_{\gamma, \mathrm{im}}\right)
\end{gathered}
$$

which allow us to rewrite (10) more compactly in the form

$$
\begin{aligned}
& Z_{\mathrm{re}}=A_{\mathrm{re}} \boldsymbol{x} \\
& Z_{\mathrm{im}}=A_{\mathrm{im}} \boldsymbol{x}
\end{aligned}
$$


where the subscripts have been dropped, $\boldsymbol{x}=\left(\begin{array}{ll}R_{\infty} & \boldsymbol{\gamma}^{\top}\end{array}\right)^{\top}$ for (13a), and $\boldsymbol{x}=\left(\begin{array}{ll}L_{0} & \boldsymbol{\gamma}^{\top}\end{array}\right)^{\top}$ for (13b).

We will suppose that a given EIS experiment is a realization of the following stochastic process:

$$
\begin{aligned}
& Z_{\mathrm{exp}, \mathrm{re}}=\boldsymbol{A}_{\mathrm{re}} \boldsymbol{x}+\boldsymbol{\varepsilon}_{\mathrm{re}} \\
& \boldsymbol{Z}_{\mathrm{exp}, \mathrm{im}}=\boldsymbol{A}_{\mathrm{im}} \boldsymbol{x}+\boldsymbol{\varepsilon}_{\mathrm{im}}
\end{aligned}
$$

where the "errors", $\varepsilon_{\mathrm{re}}$ and $\boldsymbol{\varepsilon}_{\mathrm{im}}$, are two independent Gaussian RVs, such that $\boldsymbol{\varepsilon}_{\mathrm{re}}, \boldsymbol{\varepsilon}_{\mathrm{im}} \sim \mathcal{N}\left(\mathbf{0}, \sigma_{n}^{2} \mathbf{I}\right)$ with $\mathbf{I}$ being the $M \times M$ identity matrix. In the ensuing derivation, we will drop the subscripts "re" and "im" from (14) as the two are notationally identical.

\subsubsection{Bayesian Hilbert Transform}

Bayesian methods leverage conditional probabilities. In particular, we can write that [43-45]

$$
p\left(\boldsymbol{x} \mid \boldsymbol{\omega}, \boldsymbol{Z}_{\exp }\right) p\left(\boldsymbol{Z}_{\exp } \mid \boldsymbol{\omega}\right)=p(\boldsymbol{x}) p\left(\boldsymbol{Z}_{\exp } \mid \boldsymbol{\omega}, \boldsymbol{x}\right)
$$

where $p(\cdot)$ is the pdf of the RV in the brackets, the symbol "l" indicates "conditioned to", and, again, $\boldsymbol{Z}_{\text {exp }}$ is the experimentally measured real or imaginary part of the impedance. The $p\left(\boldsymbol{Z}_{\text {exp }} \mid \boldsymbol{\omega}, \boldsymbol{x}\right)$ can be obtained from (14) as

$$
p\left(\boldsymbol{Z}_{\exp } \mid \boldsymbol{\omega}, \boldsymbol{x}\right)=p(\boldsymbol{\varepsilon}) \propto \exp \left(-\frac{1}{2 \sigma_{n}^{2}}\left\|\boldsymbol{A} \boldsymbol{x}-\boldsymbol{Z}_{\exp }\right\|^{2}\right)
$$

If we specify a prior on $\boldsymbol{x}$ (i.e. we assume $p(\boldsymbol{x})$ ), then we can obtain $p\left(\boldsymbol{x} \mid \boldsymbol{\omega}, \boldsymbol{Z}_{\text {exp }}\right)$. We will take $\boldsymbol{x} \sim \mathcal{N}\left(0, \boldsymbol{W}^{-1}\right)$, i.e.,

$$
p(\boldsymbol{x}) \propto \exp \left(-\frac{1}{2} \boldsymbol{x}^{\top} \boldsymbol{W} \boldsymbol{x}\right)
$$


with the matrix $\boldsymbol{W}$ defined as

$$
\boldsymbol{W}=\frac{1}{\sigma_{\beta}^{2}} \boldsymbol{I}+\frac{1}{\sigma_{\lambda}^{2}}\left(\begin{array}{cc}
0 & 0 \\
0 & \boldsymbol{D}_{q}^{\top} \boldsymbol{D}_{q}
\end{array}\right)
$$

where $\sigma_{\beta}^{2}$ and $\sigma_{\lambda}^{2}$ are two real numbers and $\boldsymbol{D}_{q}$ is the $q^{\text {th }}$ differentiation matrix.[40, 42]

Plugging (16) and (17) into (15) gives the posterior

$$
p\left(\boldsymbol{x} \mid \boldsymbol{\omega}, \boldsymbol{Z}_{\exp }\right) \propto \exp \left(-\frac{1}{2 \sigma_{n}^{2}}\left\|\boldsymbol{A} \boldsymbol{x}-\boldsymbol{Z}_{\exp }\right\|^{2}-\frac{1}{2} \boldsymbol{x}^{\top} \boldsymbol{W} \boldsymbol{x}\right)
$$

From the latter, it follows that

$$
\boldsymbol{x} \mid \boldsymbol{\omega}, \boldsymbol{Z}_{\mathrm{exp}} \sim \mathcal{N}\left(\boldsymbol{\mu}_{x}, \boldsymbol{\Sigma}_{x}\right)
$$

where

$$
\begin{gathered}
\boldsymbol{\mu}_{x}=\frac{1}{\sigma_{n}^{2}} \boldsymbol{\Sigma}_{x} \boldsymbol{A}^{\top} \boldsymbol{Z} \\
\boldsymbol{\Sigma}_{x}=\left(\frac{1}{\sigma_{n}^{2}} \boldsymbol{A}^{\top} \boldsymbol{A}+\boldsymbol{W}\right)^{-1}
\end{gathered}
$$

We note that $\boldsymbol{\mu}_{x}$ and $\boldsymbol{\Sigma}_{x}$ are functions of the scalars $\sigma_{n}^{2}, \sigma_{\beta}^{2}$, and $\sigma_{\lambda}^{2}$, which we will collect in the vector $\boldsymbol{\theta}=\left(\sigma_{n}^{2}, \sigma_{\beta}^{2}, \sigma_{\lambda}^{2}\right)^{\top}$.

Once the $\boldsymbol{x} \mid \boldsymbol{Z}_{\text {exp }}$ is estimated, we can use (8) to compute $Z_{\mathrm{H}}\left(\omega_{\star}\right)$, either $-H Z_{\mathrm{im}}\left(\omega_{\star}\right)$ or $H Z_{\text {re }}\left(\omega_{\star}\right)$, at a new angular frequency $\omega_{\star}$ as

$$
Z_{\mathrm{H}}\left(\omega_{\star}\right)=\boldsymbol{h} \boldsymbol{x} \sim \mathcal{N}\left(\boldsymbol{h} \boldsymbol{\mu}_{x}, \boldsymbol{h} \boldsymbol{\Sigma}_{x} \boldsymbol{h}^{\top}\right)
$$


where the column vector $\boldsymbol{h} \in \mathbb{R}^{N+1}$ is either $\boldsymbol{h}_{\mathrm{re}}$ or $\boldsymbol{h}_{\mathrm{im}}$ defined next. We note that $\left(\boldsymbol{h}_{\mathrm{re}}\right)_{1}=$ $\left(\boldsymbol{h}_{\text {im }}\right)_{1}=0$, while all other entries (for $\left.n=1,2,3, \ldots, N\right)$ are

$$
\begin{gathered}
\left(\boldsymbol{h}_{\mathrm{re}}\right)_{n+1}=\int_{-\infty}^{\infty} \psi_{\mathrm{re}}\left(\omega_{\star}, \tau\right) \phi_{n}(\log \tau) d \log \tau \\
\left(\boldsymbol{h}_{\mathrm{im}}\right)_{n+1}=\int_{-\infty}^{\infty} \psi_{\mathrm{im}}\left(\omega_{\star}, \tau\right) \phi_{n}(\log \tau) d \log \tau
\end{gathered}
$$

More precisely, for the HT of the imaginary part of the data $\left(\boldsymbol{Z}_{\text {exp }}=\boldsymbol{Z}_{\text {exp,im }} \& \boldsymbol{Z}_{\mathrm{H}}=\boldsymbol{Z}_{\mathrm{H}, \mathrm{re}}\right)$ we need to take $\boldsymbol{h}=\boldsymbol{h}_{\text {re }}$. Instead, for the HT of the real part of the data $\left(\boldsymbol{Z}_{\text {exp }}=\boldsymbol{Z}_{\text {exp,re }} \&\right.$ $\left.\boldsymbol{Z}_{\mathrm{H}}=\boldsymbol{Z}_{\mathrm{H}, \mathrm{im}}\right)$ we need to set $\boldsymbol{h}=\boldsymbol{h}_{\mathrm{im}}$.

We can rewrite (22) in matrix form as

$$
\boldsymbol{Z}_{\mathrm{H}}\left(\boldsymbol{\omega}_{\star}\right)=\boldsymbol{H} \boldsymbol{x} \sim \mathcal{N}\left(\boldsymbol{\mu}_{\mathrm{H}}, \boldsymbol{\Sigma}_{\mathrm{H}}\right)
$$

where the angular frequency vector is defined as $\boldsymbol{\omega}_{\star}=\left(\omega_{\star, 1}, \omega_{\star, 2}, \ldots, \omega_{\star, K}\right)^{\top} \in \mathbb{R}^{K}$ and the $\boldsymbol{Z}_{\mathrm{H}}\left(\boldsymbol{\omega}_{\star}\right)=\left(Z_{\mathrm{H}}\left(\omega_{\star, 1}\right), Z_{\mathrm{H}}\left(\omega_{\star, 2}\right), \ldots, Z_{\mathrm{H}}\left(\omega_{\star, K}\right)\right)^{\top} \in \mathbb{R}^{K}$. Further to that,

$$
\begin{gathered}
\boldsymbol{\mu}_{H}=\boldsymbol{H} \boldsymbol{\mu}_{x} \\
\boldsymbol{\Sigma}_{\mathrm{H}}=\boldsymbol{H} \boldsymbol{\Sigma}_{x} \boldsymbol{H}^{\top}
\end{gathered}
$$

with the following definition of the matrix $\boldsymbol{H} \in \mathbb{R}^{K \times(N+1)}$

$$
\boldsymbol{H}\left(\boldsymbol{\omega}_{\star}\right)=\left(\begin{array}{c}
\boldsymbol{h}\left(\omega_{\star, 1}\right) \\
\boldsymbol{h}\left(\omega_{\star, 2}\right) \\
\vdots \\
\boldsymbol{h}\left(\omega_{\star, K}\right)
\end{array}\right)
$$


The DRT-only part of the EIS spectrum, i.e., $Z_{\mathrm{DRT}}$, for which the resistance and inductance contributions are not considered, can be obtained at $\boldsymbol{\omega}_{\star}$ using

$$
\boldsymbol{Z}_{\mathrm{DRT}}\left(\boldsymbol{\omega}_{\star}\right)=\boldsymbol{A}_{\mathrm{DRT}} \boldsymbol{x}
$$

where the $\boldsymbol{A}_{\mathrm{DRT}} \in \mathbb{R}^{K \times(N+1)}$ is defined following an analogous reasoning and notation behind (23) and (26). In particular, we will define the entries of $\boldsymbol{A}_{\mathrm{DRT}, \mathrm{re}}$ or $\boldsymbol{A}_{\mathrm{DRT}, \text { im }}$ to be 0 in the first column, i.e. $\left(\boldsymbol{A}_{\mathrm{DRT}, \mathrm{re}}\right)_{k, 1}=\left(\boldsymbol{A}_{\mathrm{DRT}, \mathrm{im}}\right)_{k, 1}=0$, and

$$
\begin{aligned}
& \left(\boldsymbol{A}_{\mathrm{DRT}, \mathrm{re}}\right)_{k, n+1}=\int_{-\infty}^{\infty} \psi_{\mathrm{re}}\left(\left(\boldsymbol{\omega}_{\star}\right)_{k}, \tau\right) \phi_{n}(\log \tau) d \log \tau \\
& \left(\boldsymbol{A}_{\mathrm{DRT}, \mathrm{im}}\right)_{k, n+1}=\int_{-\infty}^{\infty} \psi_{\mathrm{im}}\left(\left(\boldsymbol{\omega}_{\star}\right)_{k}, \tau\right) \phi_{n}(\log \tau) d \log \tau
\end{aligned}
$$

for $k=1,2, \ldots, K$ and $n=1,2, \ldots, N$. As above $\boldsymbol{Z}_{\mathrm{DRT}}\left(\boldsymbol{\omega}_{\star}\right)$ is a multivariate gamma, i.e.,

$$
\boldsymbol{Z}_{\mathrm{DRT}}\left(\boldsymbol{\omega}_{\star}\right) \sim \mathcal{N}\left(\boldsymbol{\mu}_{\mathrm{DRT}}, \boldsymbol{\Sigma}_{\mathrm{DRT}}\right)
$$

where $\boldsymbol{\mu}_{\mathrm{DRT}}$ and $\boldsymbol{\Sigma}_{\mathrm{DRT}}$ are defined as

$$
\begin{gathered}
\boldsymbol{\mu}_{\mathrm{DRT}}=\boldsymbol{A}_{\mathrm{DRT}} \boldsymbol{\mu}_{x} \\
\boldsymbol{\Sigma}_{\mathrm{DRT}}=\boldsymbol{A}_{\mathrm{DRT}} \boldsymbol{\Sigma}_{x} \boldsymbol{A}_{\mathrm{DRT}}^{\top}
\end{gathered}
$$

\subsubsection{Choosing the Hyperparameters}

The analysis described in the previous subsection can be carried out only if the hyperparameter vector $\boldsymbol{\theta}=\left(\sigma_{n}^{2}, \sigma_{\beta}^{2}, \sigma_{\lambda}^{2}\right)^{\top}$ is set.[43, 44] To determine it, we will maximize the marginal 
likelihood (or evidence),[46] obtained by integrating (or marginalizing) the likelihood, (16), with respect to the prior:

$$
p\left(\boldsymbol{Z}_{\exp } \mid \boldsymbol{\omega}, \boldsymbol{\theta}\right)=\int_{\mathbb{R}^{N+1}} p\left(\boldsymbol{Z}_{\exp } \mid \boldsymbol{x}, \sigma_{n}^{2}\right) p\left(\boldsymbol{x} \mid \sigma_{\beta}^{2}, \sigma_{\lambda}^{2}\right) d \boldsymbol{x}
$$

As the prior is Gaussian, we can follow classical manipulations (see Appendix A) and obtain[45]

$$
\log p\left(\boldsymbol{Z}_{\exp } \mid \boldsymbol{\omega}, \boldsymbol{\theta}\right)=\frac{1}{2} \log |\boldsymbol{W}|-\frac{1}{2} \log \left|\boldsymbol{\Sigma}_{x}^{-1}\right|-\frac{M}{2} \log \left(\sigma_{n}^{2}\right)-E\left(\boldsymbol{\mu}_{x}\right)-\frac{M}{2} \log (2 \pi)
$$

In the implementation of the BHT method, the $\boldsymbol{\theta}$ maximizing the experimental evidence is found by minimizing the negative log-likelihood defined as

$$
\mathcal{L}\left(\boldsymbol{\theta}, \boldsymbol{\omega}, \boldsymbol{Z}_{\text {exp }}\right)=-\log p\left(\boldsymbol{Z}_{\exp } \mid \boldsymbol{\omega}, \boldsymbol{\theta}\right)
$$

in other words

$$
\boldsymbol{\theta}=\underset{\boldsymbol{\theta}^{\prime}}{\operatorname{argmin}} \mathcal{L}\left(\boldsymbol{\theta}^{\prime}, \boldsymbol{\omega}, \boldsymbol{Z}_{\text {exp }}\right)
$$

\subsection{Scoring the EIS Data}

In the scientific literature, there are no metrics that can be used to score the compliance of the EIS data with the KK relations. In this section, we develop four new metrics based on residuals, mean predictions, and distances between estimated distributions. All the scores were defined so that their outcomes are real numbers between 0 and 1. An HT-consistent EIS spectrum will score near 1. Instead, an HT-inconsistent EIS spectrum will score close to 0.

In the literature about KK relations applied to EIS, the quality of the impedance data is typically assessed by examining the residuals. Such residuals are obtained by subtracting (up to a constant or a linear function of the angular frequency) the experimental real/imaginary data 
from the HT estimate obtained using the imaginary/real part of the EIS data. One metric could leverage the residuals by computing the number of experimental points that lie within $k$ standard deviations of the HT prediction. Such a score can be formally defined as

$$
\begin{gathered}
s_{k \sigma, \mathrm{re}}=\frac{1}{M} \sum_{m=1}^{M} 1\left(\left|R_{\infty}+Z_{\mathrm{H}, \mathrm{re}}\left(\omega_{m}\right)-Z_{\mathrm{exp}, \mathrm{re}}\left(\omega_{m}\right)\right| \leq k \sigma\left(\omega_{m}\right)\right) \\
s_{k \sigma, \mathrm{im}}=\frac{1}{M} \sum_{m=1}^{M} 1\left(\left|\omega_{m} L_{0}+Z_{\mathrm{H}, \mathrm{re}}\left(\omega_{m}\right)-Z_{\text {exp,im }}\left(\omega_{m}\right)\right| \leq k \sigma\left(\omega_{m}\right)\right)
\end{gathered}
$$

where $1(\cdot)$ is the indicator function, which is 1 if its argument is true and 0 otherwise, $\sigma\left(\omega_{m}\right)$ is the standard deviation of the HT $\omega_{m}$, and $R_{\infty}$ and $L_{0}$ are obtained by Bayesian regression from $Z_{\text {exp,re }}$ and $Z_{\text {exp,im }}$, respectively. We note that $0 \leq s_{k \sigma} \leq 1$ and $s_{k \sigma} \leq s_{(k+1) \sigma}$ and that the smaller the residual the closer will the score be to 1 .

The $s_{k \sigma, \text { re }}$ and $s_{k \sigma, \text {,im }}$ metrics compare data, the realization of an $\mathrm{RV}$, and predictions, other RVs. However, other useful scores could compare the two de facto analogous RVs, that can be obtained from different parts of the data, namely $\boldsymbol{Z}_{\mathrm{H}}(\boldsymbol{\omega}) \sim \mathcal{N}\left(\boldsymbol{\mu}_{\mathrm{H}}, \boldsymbol{\Sigma}_{\mathrm{H}}\right)$ and $\boldsymbol{Z}_{\mathrm{DRT}}(\boldsymbol{\omega}) \sim \mathcal{N}\left(\boldsymbol{\mu}_{\mathrm{DRT}}, \boldsymbol{\Sigma}_{\mathrm{DRT}}\right)$. One proposed score, which we call $s_{\mu}$, consists in summing 1 to the negative of the relative distance between the mean vectors $\boldsymbol{\mu}_{\mathrm{DRT}}$ and $\boldsymbol{\mu}_{\mathrm{H}}$ :

$$
\begin{gathered}
s_{\mu, \mathrm{re}}=1-\frac{\left\|\boldsymbol{\mu}_{\mathrm{DRT}, \mathrm{re}}-\boldsymbol{\mu}_{\mathrm{H}, \mathrm{re}}\right\|}{\left\|\boldsymbol{\mu}_{\mathrm{DRT}, \mathrm{re}}\right\|+\left\|\boldsymbol{\mu}_{\mathrm{H}, \mathrm{re}}\right\|} \\
s_{\mu, \mathrm{im}}=1-\frac{\left\|\boldsymbol{\mu}_{\mathrm{DRT}, \mathrm{im}}-\boldsymbol{\mu}_{\mathrm{H}, \mathrm{im}}\right\|}{\left\|\boldsymbol{\mu}_{\mathrm{DRT}, \mathrm{im}}\right\|+\left\|\boldsymbol{\mu}_{\mathrm{H}, \mathrm{im}}\right\|}
\end{gathered}
$$

From these last two formulas, it follows that $0 \leq s_{\mu} \leq 1$ and that the closer is $s_{\mu}$ to the 1 , the more similar will be the two means $\boldsymbol{\mu}_{\mathrm{DRT}}$ and $\boldsymbol{\mu}_{\mathrm{H}}$. 
Another sensible approach entails comparing the pdf's of $\boldsymbol{Z}_{\mathrm{H}}(\boldsymbol{\omega})$ and $\boldsymbol{Z}_{\mathrm{DRT}}(\boldsymbol{\omega})$. This can be done using a number of metrics. One particularly convenient one is called Hellinger distance (HD).[47] The square of the $\operatorname{HD},(\operatorname{HD}(f, g))^{2}$, between two pdfs $f(x)$ and $g(x)$ can be defined as

$$
(\mathrm{HD}(f, g))^{2}=1-\int \sqrt{f(x) g(x)} d x
$$

where $0 \leq \operatorname{HD}(f, g) \leq 1$ and $\operatorname{HD}(f, g)=0$ if and only if $f(x)=g(x)$. Therefore, a viable score could use[47]

$$
\left(\mathrm{HD}\left(p_{Z_{\mathrm{DRT}}}, p_{Z_{\mathrm{H}}}\right)\left(\omega_{\star}\right)\right)^{2}=1-\sqrt{\frac{2 \sigma_{\mathrm{DRT}} \sigma_{\mathrm{H}}}{\sigma_{\mathrm{DRT}}^{2}+\sigma_{\mathrm{H}}^{2}}} e^{-\frac{1\left(\mu_{\mathrm{DRT}}-\mu_{\mathrm{H}}\right)^{2}}{4 \sigma_{\mathrm{DRT}}^{2}+\sigma_{\mathrm{H}}^{2}}}
$$

where $p_{Z_{\mathrm{DRT}}}$ and $p_{Z_{\mathrm{H}}}$ are the pdfs of $Z_{\mathrm{DRT}}\left(\omega_{\star}\right)$ and $Z_{\mathrm{H}}\left(\omega_{\star}\right)$ at the scalar angular frequency $\omega_{\star}$. We must point out that in (38) the dependence of $\mu_{\mathrm{DRT}}, \sigma_{\mathrm{DRT}}, \mu_{\mathrm{H}}$, and $\sigma_{\mathrm{H}}$ on $\omega_{\star}$ has been omitted for the sake of keeping the equation compact. Similar to what we did for the residuals, we can define an average HD as follows

$$
\overline{\mathrm{HD}}=\frac{1}{M} \sum_{m=1}^{M} \operatorname{HD}\left(p_{Z_{\mathrm{DRT}}}, p_{Z_{\mathrm{H}}}\right)\left(\omega_{m}\right)
$$

which allows us to compute two HD scores, $s_{\mathrm{HD}, \text { re }}$ and $s_{\mathrm{HD} \text {,im }}$

$$
\begin{aligned}
& s_{\mathrm{HD}, \mathrm{re}}=1-\overline{\mathrm{HD}}_{\mathrm{re}} \\
& s_{\mathrm{HD}, \mathrm{im}}=1-\overline{\mathrm{HD}}_{\mathrm{im}}
\end{aligned}
$$

where $\overline{\mathrm{HD}}_{\mathrm{re}}$ is the average Hellinger distance between $Z_{\mathrm{DRT} \text {,re }}$ and $Z_{\mathrm{H}, \mathrm{re}}$ over $\omega_{\star}$ and $\overline{\mathrm{HD}}_{\mathrm{im}}$ is the average Hellinger distance between $Z_{\mathrm{DRT}, \mathrm{im}}$ and $Z_{\mathrm{H}, \mathrm{im}}$ over $\omega_{\star}$. We should stress that while 
$Z_{\mathrm{DRT}, \mathrm{re}}$ and $Z_{\mathrm{H}, \mathrm{im}}$ are obtained from the sole real part of the EIS spectrum, $Z_{\mathrm{DRT}, \mathrm{im}}$ and $Z_{\mathrm{H}, \mathrm{re}}$ are calculated using only the imaginary portion of the EIS. Therefore, both $\overline{\mathrm{HD}}_{\mathrm{re}}$ and $\overline{\mathrm{HD}}_{\mathrm{im}}$ compare the real with the imaginary part of the EIS data.

A final metric leverages the Kullback-Leibler (KL) discrepancy or relative entropy, $d_{\mathrm{KL}}(\cdot \| \cdot)$, which is often used to measure the degree of similarity between the two pdfs.[45] Unfortunately, we were not able to produce a consistent score using the KL divergence alone, because $d_{\mathrm{KL}}(\cdot \| \cdot)$ is neither symmetric nor bounded. Instead, we will use the Jensen-Shannon divergence (JSD), which is based on the KL but is symmetric and bounded between 0 and $\log 2 .[48]$ If we define

$$
Z_{\mathrm{M}}\left(\omega_{\star}\right)=\frac{1}{2}\left(Z_{\mathrm{DRT}}\left(\omega_{\star}\right)+Z_{\mathrm{H}}\left(\omega_{\star}\right)\right)
$$

the JSD between $Z_{\mathrm{DRT}}\left(\omega_{\star}\right)$ and $Z_{\mathrm{H}}\left(\omega_{\star}\right)$ is the following symmetrized KL divergence:

$$
\operatorname{JSD}\left(Z_{\mathrm{DRT}}\left(\omega_{\star}\right), Z_{\mathrm{H}}\left(\omega_{\star}\right)\right)=\frac{1}{2}\left(d_{\mathrm{KL}}\left(Z_{\mathrm{DRT}}\left(\omega_{\star}\right) \| Z_{\mathrm{M}}\left(\omega_{\star}\right)\right)+d_{\mathrm{KL}}\left(Z_{\mathrm{H}}\left(\omega_{\star}\right) \| Z_{\mathrm{M}}\left(\omega_{\star}\right)\right)\right)
$$

As we did above for residuals and HD, the average JSD will be averaged over the experiment as follows:

$$
\overline{\mathrm{JSD}}\left(Z_{\mathrm{DRT}}, Z_{\mathrm{H}}\right)=\frac{1}{2 M} \sum_{m=1}^{M}\left(d_{\mathrm{KL}}\left(Z_{\mathrm{DRT}}\left(\omega_{m}\right) \| Z_{\mathrm{M}}\left(\omega_{m}\right)\right)+d_{\mathrm{KL}}\left(Z_{\mathrm{H}}\left(\omega_{m}\right) \| Z_{\mathrm{M}}\left(\omega_{m}\right)\right)\right)
$$

Therefore, we can develop two JSD scores

$$
s_{\mathrm{JSD}, \mathrm{re}}=\frac{\log 2-\overline{\mathrm{JSD}}_{\mathrm{re}}}{\log 2}
$$




$$
s_{\mathrm{JSD}, \mathrm{im}}=\frac{\log 2-\overline{\mathrm{JSD}}_{\mathrm{im}}}{\log 2}
$$

where $\overline{\mathrm{JSD}}_{\mathrm{re}}$ is the average JSD between $Z_{\mathrm{DRT}}$,re and $Z_{\mathrm{H}, \mathrm{re}}$ and $\overline{\mathrm{JSD}}_{\mathrm{im}}$ is the average JSD between $Z_{\mathrm{DRT} \text {,im }}$ and $Z_{\mathrm{H}, \mathrm{im}}$. Consistently with what we did for other scores, the $s_{\mathrm{JSD}}$ 's are bounded between 0 and 1 and a $S_{\text {JSD }}$ close to 1 implies that $Z_{\mathrm{DRT}}$ and $Z_{\mathrm{H}}$ are consistent, while if the $S_{\mathrm{JSD}}$ nears 0 , the two are inconsistent. We must note that no close formula is known for the JSD, therefore the $\overline{\mathrm{JSD}}$ was obtained by Monte Carlo sampling.

\section{Results}

\subsection{Synthetic Experiments}

To benchmark the performance of the BHT method, we tested it systematically against controlled synthetic experiments with known standard circuits. In this section, we will illustrate the BHT framework and the EIS data scoring. The BHT method follows the logical path shown in Figure 1. All circuits studied are reported in Table 1 with their parameters listed in Table 2. Consistently with our earlier articles, the frequency range of analysis was selected to be between $10^{-4}$ and $10^{4} \mathrm{~Hz}$ with a resolution of 10 points per decade.[49, 50] We must stress that the experimental impedance is generated by adding white noise to the real and imaginary parts of the exact impedance, consistently with (14) and (16). That is, known the exact circuit impedance, $Z_{\text {exact }}(\omega)$, the experimental data $Z_{\exp }(\omega)$ is obtained as follows:

$$
Z_{\text {exp }}(\omega)=Z_{\text {exact }}(\omega)+\varepsilon_{\mathrm{re}}+i \varepsilon_{\mathrm{im}}
$$

where $\varepsilon_{\mathrm{re}}$ and $\varepsilon_{\mathrm{im}}$ are independent and identically distributed RVs with a Gaussian distribution

of mean 0 and standard deviation $\sigma_{n \text {,exp }}$, i.e., $\varepsilon_{\mathrm{re}}, \varepsilon_{\mathrm{im}} \sim \mathcal{N}\left(0, \sigma_{n, \exp }^{2}\right)$. We note that $\sigma_{n, \exp }$ is 
conceptually different from $\sigma_{n}$. While $\sigma_{n \text {,exp }}$ is a parameter used when drawing the synthetic experimental data, $\sigma_{n}$ is one of the hyperparameters that need to be optimized by maximizing the evidence during the analysis. In all stochastic experiments, we will set $\sigma_{n \text {,exp }}=0.8 \sqrt{\Omega}$, a relatively high value for the given circuit parameters.

As a first step, we investigated how the BHT performs for a resistor in series with a ZARC element. The corresponding synthetic impedance spectrum obtained using (45) is shown in Figure 2. The real and imaginary parts can be separated and regressed separately using (20) to obtain the latent $\boldsymbol{x}$. In turn, $\boldsymbol{x}$ can be used to obtain 1) the regressed and smoothed impedance, $Z ; 2$ ) the $R_{0}$ - and $L_{0}$-subtracted impedance, $Z_{\mathrm{DRT}}$; and 3) the HT impedance, $Z_{\mathrm{H}}$. The real and imaginary parts of the experimental EIS are shown in Figure 2 (b) and (c). In these two panels, the mean impedance (black line) and the $3 \sigma$ credible intervals (solid grey shading) are reported. By visual inspection, it appears that the stochastic experiments fall within the credible bands. As already outlined above, the hyperparameter vector $\boldsymbol{\theta}=\left(\sigma_{n}^{2}, \sigma_{\beta}^{2}, \sigma_{\lambda}^{2}\right)^{\top}$ used to find these estimates was obtained by evidence maximization, which was achieved by finding the minimum of $\mathcal{L}\left(\boldsymbol{\theta}, \boldsymbol{\omega}, \boldsymbol{Z}_{\text {exp }}\right)$ with respect to $\boldsymbol{\theta}$. We plotted in Figure 3 the $\mathcal{L}\left(\boldsymbol{\theta}, \boldsymbol{\omega}, \boldsymbol{Z}_{\text {exp,re }}\right)$, panel (a), and $\mathcal{L}\left(\boldsymbol{\theta}, \boldsymbol{\omega}, \boldsymbol{Z}_{\text {exp,im }}\right)$ as a function of $\sigma_{\beta}$ and $\sigma_{\lambda}$, where in both cases the $\sigma_{n}$ was fixed, i.e., $\sigma_{n}=\sigma_{n, \text { exp }}$. We can see that the minimum is rather sharp and elongated along the $\sigma_{\lambda}$ direction, indicating a small penalty on the derivative. Interestingly, for both real and imaginary data, the minima are obtained for relatively small values of $\frac{1}{\sigma_{\beta}^{2}}$ and $\frac{1}{\sigma_{\lambda}^{2}}\left(\frac{1}{\sigma_{\beta}^{2}}=2.93 \times 10^{-3}\right.$ and $7.36 \times 10^{-3}$ and $\frac{1}{\sigma_{\lambda}^{2}}=1.36 \times 10^{-3}$ and $1.36 \times 10^{-3}$ for the real and imaginary parts, respectively), which correspond to a small smoothing penalty, as realized by the matrix $\boldsymbol{W}$, on the priorless term $\frac{1}{\sigma_{n}^{2}} \boldsymbol{A}^{\top} \boldsymbol{A}$. After conducting the Bayesian regression, $Z_{\mathrm{H}}$ and $Z_{\mathrm{DRT}}$ can be predicted. In 
particular, upon adding the $R_{\infty}$ or $\omega L_{0}$, the $Z_{\mathrm{H}}$ can be compared to the experiments. The comparison is reported in Figure 4 (a) and (b) for the real and imaginary parts of the EIS, respectively. It is apparent that $Z_{\mathrm{H}}$ as obtained from the imaginary (real) part can well recover the real (imaginary) part of the stochastic experiment. This is further confirmed by the $s_{\mu}$ values, which are in the range of $0.95-0.98$, see Table 3 . An alternative view is given by the residuals, which are obtained by subtracting the experimental data from the mean HT's impedance, $\boldsymbol{\mu}_{\mathrm{H}}$, plus the offset $R_{\infty}$ or $\omega L_{0}$. Figure 4 (c) and (d) report the real and imaginary residuals, respectively, together with their $3 \sigma$ bands (solid gray region). We can observe that all points fall within the grey region, indicating that $s_{3 \sigma \text {,re }}=s_{3 \sigma \text {,im }}=1$. From the scores reported in Table 3, the residual scores are high suggesting that the EIS spectrum is HT- (or KK-) consistent. However, we note that a visual inspection of the residuals does not yield any direct information about the distribution of the residuals. Therefore, we also reported the kernel-estimated densities of the residuals in Figure 4 (e) and (f). There, we observe that the densities are centered around 0 and endowed with a high degree of symmetry. Further, as one can see from Table 3 , the $s_{\mu}, s_{\mathrm{HD}}$, and $s_{\mathrm{JSD}}$ are closer to 1 rather than 0 , suggesting that the estimated distributions of $Z_{\mathrm{H}}$ and $Z_{\mathrm{DRT}}$ also match one another.

We then analyzed two sets of synthetic experiments based on two ZARCs in series, see Table 1 and Table 2 for the exact impedance and parameter values, respectively. For these circuits, it is well known that the latent $\gamma$ cannot be obtained easily, especially if the smoothing (i.e. $\left.\frac{1}{\sigma_{\lambda}^{2}}\right)$ is too strong and the characteristic timescales are partially or fully overlapping.[43, 44] Figure 5 (a) and (b) report the exact and stochastic EIS spectra of the two cases. Figure 5 (c) and (d) correspond to the regressed imaginary parts. We note that similar regression was also done for the real part, see Table 3, but is not shown for the sake of brevity. Before discussing Figure 6, 
we must point out that the panels (a) \& (c) and (b) \& (d) of Figure 5 correspond to (a), (c) \& (e) and (b), (d) \& (f) in Figure 6. The obtained HT's impedances, $Z_{\mathrm{H}}$, are plotted in Figure 6 (a) and (b). Both $Z_{\mathrm{H}}$ match well the synthetic experiment, leading to $s_{\mu} \approx 1$. Similarly, we plotted residuals and their distributions in Figure 6 (c) \& (e) and (d) \& (f). Unsurprisingly, the results are consistent with expectations that the residuals should fall within the $3 \sigma$ bands with rather symmetric distributions. As shown in Table 3, all scores confirm the built-in consistency of the EIS spectrum.

We also applied the BHT method to the piecewise constant (PWC) element. This element is somewhat pathological because the exact $\gamma$ is discontinuous and therefore requires ad hoc regularization.[43, 44] We report in Figure 7 (a) the exact and stochastic EIS of the PWC element and in Figure 7 (b) the regressed imaginary part. The $Z_{\mathrm{H}, \mathrm{im}}$, reported in Figure 7 (c), which was obtained with the data of Figure 7 (b), matches the experiment well. Furthermore, the residuals, shown in Figure 7 (d), appear to be well within the $3 \sigma$ credible interval with the underlying distribution being characterized by some degree of symmetry. The scores, see Table 3, are also consistent with the visual analysis and indicate, as expected, that the data complies with the HT.

The stochastic experiments illustrated above assumed the presence of only a resistor connected in series to the main elements. However, inductive features can also be present in the EIS data. These inductances can be modeled by adding a $\omega L_{0}$ term to the imaginary part of the impedance, see (5). While the HT of a constant is formally 0 , the HT of $\omega L_{0}$ is infinite.[31] Therefore, the inductance component needs to be subtracted from the impedance data at the regression stage and added later. This is a delicate issue that the BHT method can handle. We show that in Figure 8 (a), where an inductor is added to the circuit of Figure 2 (a), see Table 2 
for the parameter values. Our framework can not only recover the imaginary part of the impedance, as shown in Figure 8 (b), but also reliably retrieve an HT of the data that matches the experiments well. This consistency is shown in the prediction and residual plots in Figure 8 (c) and (d), respectively, and in all computed scores, see Table 3. One may also wonder how well the BHT does in retrieving the mean values of the circuit parameters, i.e., $R_{\infty}$ and $L_{0}$, as and in retrieving the $\sigma_{n, \exp }$ from $\boldsymbol{\theta}$. We tested that by carrying out 2000 stochastic experiments, for which the $\boldsymbol{\theta}$ 's were obtained by maximizing the evidence. The resulting mean values of $R_{\infty}$ and $L_{0}$ and the corresponding $\sigma_{n}=(\boldsymbol{\theta})_{1}$ are shown in Figure 9 as a joint distribution plot. The values obtained deviate little from their externally assigned values, see Table 2, showing little correlation to one another. One important point to note is that, while using these point estimates does not comply with the Bayesian philosophy, we think it is important to highlight that the BHT methodology developed here consistently retrieves the ground truth of the studied synthetic experiments.

As a last stochastic experiment, we wish to determine if the BHT can detect inconsistent impedance data. To this end, we use a circuit composed of two slightly different transfer functions for the real and imaginary parts. The actual model is shown in the last row of Table 1, and the parameters can be found in Table 2. The Nyquist plot of such an artificial impedance is shown in Figure 10 (a). In this specific case, the exact impedance is not a transfer function, and therefore, the HT is expected to detect that. In other words, if we perform HT on $Z_{\mathrm{re}}(\omega)$, we will not be able to obtain $Z_{\text {im }}(\omega)$ and vice versa. The inconsistency of the data is not apparent if the real and imaginary parts of the EIS are regressed using the BHT, see Figure 10 (a) and (b). On the other hand, the HTs in Figure 11 (a) and (b) show a significant deviation from the experimental data. This discrepancy is even more evident if one looks at the residuals, which are bimodally distributed, see Figure 11 (c) and (d). Further, all scores in Table 3 are 
lower than that of the previous synthetic experiments, indicating a decreased reliability and inconsistency in the data.

\subsection{Real Experiments}

The analysis of the BHT method would be incomplete if real experiments were not analyzed. For this reason, we examined two sets of experimental data that had been used in our prior publications.[42-44] First, we tested the BHT against the data obtained from a commercial battery. $[42,44]$ The EIS spectrum was collected from a $\mathrm{LiCoO}_{2}$ battery (Ansmann 18650) at a $25 \%$ state of charge from $5 \mathrm{mHz}$ to $600 \mathrm{~Hz}$ with 10 points per decade. The impedance spectrum is drawn in Figure 12 (a). We first estimated the imaginary part of the spectrum using Bayesian regression. The estimated impedance data shows little deviation from the experimental measurement and falls within an extremely narrow credible band, see Figure 12 (b). For the obtained $Z_{\mathrm{H}, \mathrm{re}}$, the credible band only slightly enlarges to be observable and the deviations are still small, see Figure 12 (c). We further plotted the discrepancy between $Z_{\mathrm{H}}$ and $Z_{\text {exp }}$ in Figure 12 (d), where clear deviations appear only at low frequencies for $f \leq 10^{-2} \mathrm{~Hz}$. The distribution of residuals reflects this insight and is centered at the origin with small dissymmetric fringes around it. The scores in Table 3 also support the above assessment and suggest that the quality of the imaginary part of the spectrum is likely to be better than that of the real part.

Lastly, we analyzed an EIS spectrum obtained from a symmetrical solid oxide fuel cell (SOFC) with $15 \%$ Sm-doped $\mathrm{CeO}_{2}$ (SDC) as the electrolyte and $\mathrm{Ba}_{0.95} \mathrm{Laa}_{0.05} \mathrm{Fe}_{0.95} \mathrm{P}_{0.05} \mathrm{O}_{3-\delta}(\mathrm{BLFP})$ as the electrode.[51] The impedance data were obtained at $700{ }^{\circ} \mathrm{C}$ under a synthetic air atmosphere (a mixture of $\mathrm{N}_{2}: \mathrm{O}_{2}$ in a 0.79:0.21 ratio) and a total pressure of $1 \mathrm{~atm}$. The EIS measurement was conducted in a frequency ranging from $0.1 \mathrm{~Hz}$ to $2.47 \times 10^{4} \mathrm{~Hz}$ with 15 points 
per decade, as reported in Figure 13 (a). While we performed BHT regression for both the real and imaginary part of the experimental data, we only show the analysis of $Z_{\text {exp,im. }}$. As shown in Figure 13 (b), Bayesian regression is capable of capturing the imaginary part well with no notable discrepancy and a credible band too narrow to be observable. The high quality of the data is evident from Figure 13 (d), where a remarkably good match is observed between the $R_{\infty}+Z_{\mathrm{H}, \mathrm{re}}$ and the $Z_{\text {exp,im. }}$. We further show the residual between these two terms in Figure 13 (d). One can notice that the residual points are unimodally distributed with the center of the distribution placed near the origin. The high quality of the impedance data is further confirmed by the scores listed in Table 3, which indicate strong compliance with the HT.

\section{Conclusions}

Compliance with the HT or the KK relations is a cornerstone of EIS analysis. While this is an important and generally appreciated fact, practitioners rarely test their data for that. In this article, we aim at bridging this gap by putting forward two innovations. First, we reframed the HT in a probabilistic Bayesian framework. Doing so allowed us to identify the credibility of the HT'd EIS data and, in turn, use the credibility to compare predictions. Second, we established several criteria to score the impedance data. We assigned the score value between 0 and 1 , where the higher the score is, the better is the experimental impedance $Z(\omega)$ compliance with the HT. The scores were developed leveraging residuals, distances between means, as well as probabilistic discrepancies between predictions.

Looking forward, various research topics could expand on this work. The basis set used here could be further extended to include spectral and pseudo-spectral elements, e.g., radial-basis functions, to improve accuracy. Also, more metrics may be developed to score the quality of EIS against the KK relation or HT. Furthermore, new standardized test cases and procedures 
may be proposed to benchmark algorithms that assess the compliance of EIS data with the HT or KK relations.

Sharing our source code may prove to be useful to researchers and speed up innovations in this area. We also added related code to the DRTtools with the intent of releasing an easy-to-use HT package to the entire electrochemical community. Lastly, we wish to emphasize that by formalizing a BHT method, this article will likely revive research in the area and promote the benchmarking of the EIS data consistency by HT or KK relations. 


\section{Acknowledgments}

The authors acknowledge the support from the Research Grants Council of Hong Kong

(projects 16227016, and 16204517), the Hong Kong Innovation and Technology Fund (ITS/292/18FP), and the Guangzhou Science and Technology Program (No. 201807010074). 


\section{List of abbreviations and symbols}

\begin{tabular}{|c|c|}
\hline BHT & Bayesian Hilbert transform \\
\hline DRT & Distribution of relaxation times \\
\hline EIS & Electrochemical impedance spectroscopy \\
\hline HT & Hilbert transform \\
\hline HD & Hellinger distance \\
\hline JSD & Jensen-Shannon divergence \\
\hline KK & Kramers-Kronig \\
\hline KL & Kullback-Leibler \\
\hline pdf & Probability distribution function \\
\hline $\mathrm{RV}$ & Random variable \\
\hline SOFC & Solid oxide fuel cell \\
\hline $\boldsymbol{A}$ & Matrix used to regress the experimental data, $\boldsymbol{Z}_{\mathrm{exp}}$ \\
\hline $\boldsymbol{A}_{\mathrm{DRT}}$ & Matrix used to calculate $\boldsymbol{Z}_{\mathrm{DRT}}$ \\
\hline $\boldsymbol{D}_{q}$ & Differentiation matrix ( $q^{\text {th }}$ order) \\
\hline$f$ & Frequency \\
\hline $\boldsymbol{h}$ & Column vector used to compute $Z_{\mathrm{H}}$ \\
\hline $\boldsymbol{H}$ & Matrix used to compute $\boldsymbol{Z}_{\mathrm{H}}$ \\
\hline I & Identity matrix \\
\hline$L_{0}$ & Inductance \\
\hline $\mathcal{L}\left(\boldsymbol{\theta}, \boldsymbol{\omega}, \boldsymbol{Z}_{\mathrm{exp}}\right)$ & Negative log-likelihood \\
\hline$p(\cdot)$ & Probability distribution function \\
\hline$R_{\infty}$ & Ohmic resistance \\
\hline$s_{k \sigma}$ & Score based on the residuals \\
\hline$s_{\mu}$ & Score based on the estimated means \\
\hline$s_{\mathrm{HD}}$ & Score based on the Hellinger distance score \\
\hline$s_{\text {ISD }}$ & Score based on the Jensen-Shannon divergence \\
\hline
\end{tabular}




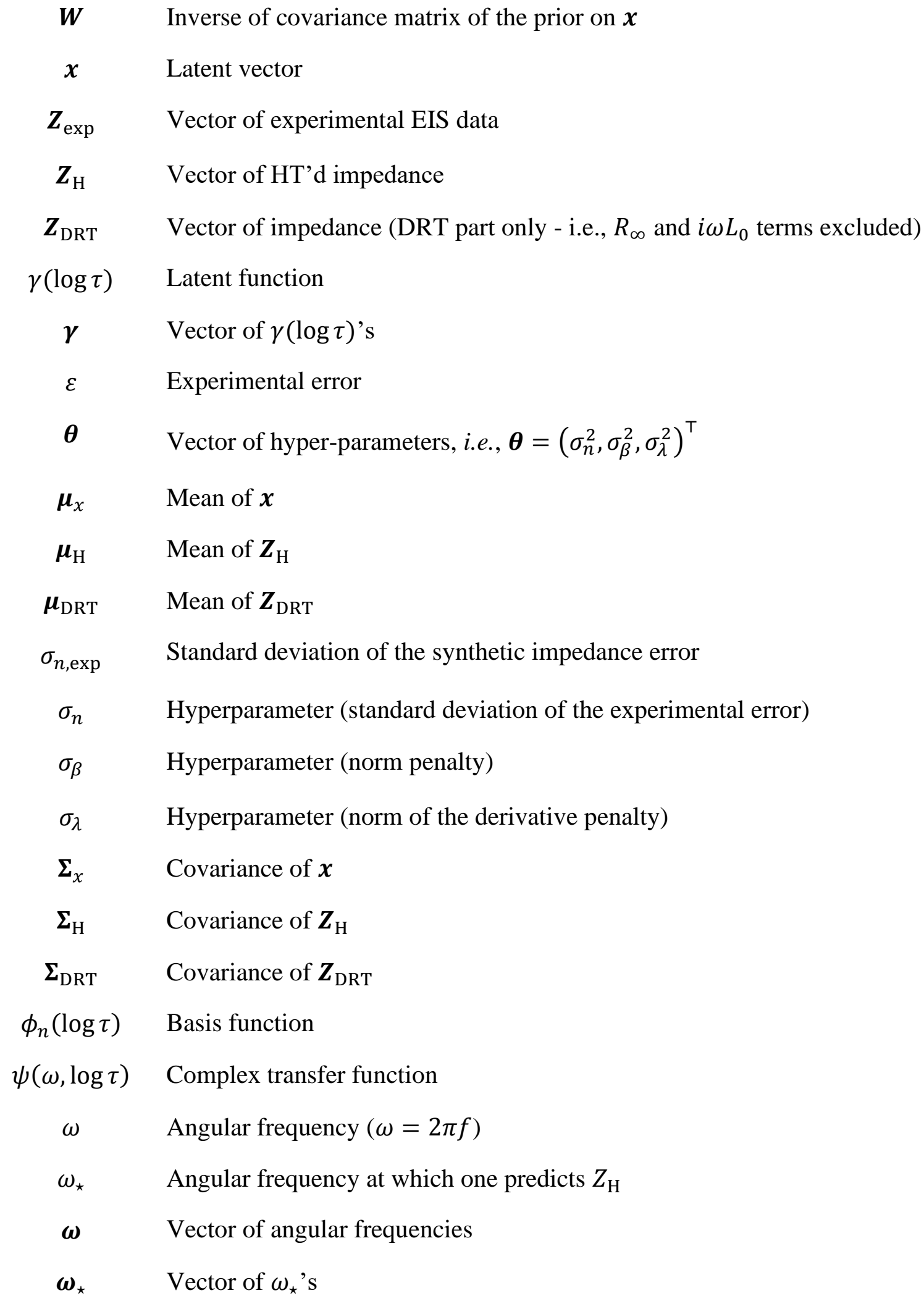




\section{Algorithm}

Input: $\quad \boldsymbol{Z}_{\text {exp,re }}$ and $\boldsymbol{Z}_{\text {exp,im }}$

Output: $\quad \boldsymbol{Z}_{\mathrm{re}}, \boldsymbol{Z}_{\mathrm{im}}, \boldsymbol{Z}_{\mathrm{H}, \mathrm{re}}, \boldsymbol{Z}_{\mathrm{H}, \mathrm{im}}, \boldsymbol{Z}_{\mathrm{DRT}, \mathrm{re}}$, and $\boldsymbol{Z}_{\mathrm{DRT}, \mathrm{im}}$

for $\boldsymbol{Z}_{\text {exp }}$ in $\left\{\boldsymbol{Z}_{\text {exp,re }}, \boldsymbol{Z}_{\text {exp,im }}\right\}$ :

1. Compute optimal $\boldsymbol{\theta}=\left(\sigma_{n}^{2}, \sigma_{\beta}^{2}, \sigma_{\lambda}^{2}\right)^{\top}$ using (33)

2. Compute optimal $\boldsymbol{\theta}=\left(\sigma_{n}^{2}, \sigma_{\beta}^{2}, \sigma_{\lambda}^{2}\right)^{\top}$ using (33)

3. Estimate $\boldsymbol{\mu}_{x}$ and $\boldsymbol{\Sigma}_{x}$ using (21)

4. Compute $\boldsymbol{\mu}_{\mathrm{H}}$ and $\boldsymbol{\Sigma}_{\mathrm{H}}$ using (25), $\boldsymbol{\mu}_{\mathrm{DRT}}$ and $\boldsymbol{\Sigma}_{\mathrm{DRT}}$ using (29)

end

compute the scores $s_{k \sigma}, s_{\mu}, s_{\mathrm{HD}}$, and $s_{\mathrm{JSD}}$.

plot: $\quad$ a. $\boldsymbol{Z}_{\mathrm{H}, \mathrm{re}}+R_{\infty}$ and $\boldsymbol{Z}_{\mathrm{H}, \mathrm{im}}+i \omega L_{0}$ against $\boldsymbol{Z}_{\text {exp,re }}$ and $\boldsymbol{Z}_{\text {exp,im }}$, respectively

b. residuals and density distribution 


\section{References}

[1] M.E. Orazem, B. Tribollet, Electrochemical impedance spectroscopy, $2^{\text {nd }}$ ed., Wiley Interscience (2017).

[2] T.P. Heins, N. Schlüter, U. Schröder, Electrode-resolved monitoring of the ageing of largescale lithium-Ion cells by using electrochemical impedance spectroscopy, ChemElectroChem, 4 (2017) 2921-2927.

[3] C. Shu, C. Wu, J. Long, H. Guo, S.-X. Dou, J. Wang, Highly reversible Li-O 2 battery induced by modulating local electronic structure via synergistic interfacial interaction between ruthenium nanoparticles and hierarchically porous carbon, Nano Energy, 57 (2019) 166-175.

[4] B.A. Boukamp, Electrochemical impedance spectroscopy in solid state ionics: recent advances, Solid State Ionics, 169 (2004) 65-73.

[5] A.K. Baral, Y. Tsur, Sintering aid ( $\mathrm{ZnO})$ effect on proton transport in $\mathrm{BaCe}_{0.35} \mathrm{Zr}_{0.5} \mathrm{Y}_{0.15} \mathrm{O}_{3-\delta}$ and electrode phenomena studied by distribution function of relaxation times, Journal of the American Ceramic Society, 102 (2019) 239-250.

[6] B. Stoeckl, V. Subotić, D. Reichholf, H. Schroettner, C. Hochenauer, Extensive analysis of large planar SOFC: operation with humidified methane and carbon monoxide to examine carbon deposition based degradation, Electrochimica Acta, 256 (2017) 325-336.

[7] A. Mroziński, S. Molin, J. Karczewski, T. Miruszewski, P. Jasiński, Electrochemical properties of porous $\mathrm{Sr}_{0.86} \mathrm{Ti}_{0.65} \mathrm{Fe}_{0.35} \mathrm{O}_{3}$ oxygen electrodes in solid oxide cells: Impedance study of symmetrical electrodes, International Journal of Hydrogen Energy, 44 (2019) 1827-1838.

[8] E. Pikalova, A. Kolchugin, M. Koroleva, G. Vdovin, A. Farlenkov, D. Medvedev, Functionality of an oxygen $\mathrm{Ca}_{3} \mathrm{Co}_{4} \mathrm{O}_{9+\delta}$ electrode for reversible solid oxide electrochemical cells based on proton-conducting electrolytes, Journal of Power Sources, 438 (2019) 226996. [9] A. Sacco, Electrochemical impedance spectroscopy: Fundamentals and application in dyesensitized solar cells, Renewable and Sustainable Energy Reviews, 79 (2017) 814-829.

[10] E. von Hauff, Impedance spectroscopy for emerging photovoltaics, The Journal of Physical Chemistry C, 123 (2019) 11329-11346.

[11] S.J. Cooper, A. Bertei, D.P. Finegan, N.P. Brandon, Simulated impedance of diffusion in porous media, Electrochimica Acta, 251 (2017) 681-689. 
[12] R. Pauliukaite, M.E. Ghica, O. Fatibello-Filho, C.M.A. Brett, Electrochemical impedance studies of chitosan-modified electrodes for application in electrochemical sensors and biosensors, Electrochimica Acta, 55 (2010) 6239-6247.

[13] R. Maalouf, C. Fournier-Wirth, J. Coste, H. Chebib, Y. Saïkali, O. Vittori, A. Errachid, J.-P. Cloarec, C. Martelet, N. Jaffrezic-Renault, Label-Free detection of bacteria by electrochemical impedance spectroscopy: comparison to surface plasmon resonance, Analytical Chemistry, 79 (2007) 4879-4886.

[14] K. Siuzdak, P. Niedziałkowski, M. Sobaszek, T. Łęga, M. Sawczak, E. Czaczyk, K. Dziąbowska, T. Ossowski, D. Nidzworski, R. Bogdanowicz, Biomolecular influenza virus detection based on the electrochemical impedance spectroscopy using the nanocrystalline boron-doped diamond electrodes with covalently bound antibodies, Sensors and Actuators B: Chemical, 280 (2019) 263-271.

[15] B.H. Brown, P. Milnes, S. Abdul, J.A. Tidy, Detection of cervical intraepithelial neoplasia using impedance spectroscopy: a prospective study, BJOG: An International Journal of Obstetrics \& Gynaecology, 112 (2005) 802-806.

[16] P.W. Weijenborg, W.O.A. Rohof, L.M.A. Akkermans, J. Verheij, A.J.P.M. Smout, A.J. Bredenoord, Electrical tissue impedance spectroscopy: a novel device to measure esophageal mucosal integrity changes during endoscopy, Neurogastroenterology \& Motility, 25 (2013) 574-e458.

[17] F. Ciucci, Modeling electrochemical impedance spectroscopy, Current Opinion in Electrochemistry, 13 (2019) 132-139.

[18] M.R.S. Urquidi-Macdonald, D.D. Macdonald, Applications of s-Kronig transforms in the analysis of electrochemical impedance data-III. Stability and linearity, Electrochimica Acta, 35 (1990) 1559-1566.

[19] M. Schönleber, D. Klotz, E. Ivers-Tiffée, A method for improving the robustness of linear Kramers-Kronig validity tests, Electrochimica Acta, 131 (2014) 20-27.

[20] E. Van Gheem, R. Pintelon, J. Vereecken, J. Schoukens, A. Hubin, P. Verboven, O. Blajiev, Electrochemical impedance spectroscopy in the presence of non-linear distortions and nonstationary behaviour: Part I: theory and validation, Electrochimica Acta, 49 (2004) 4753-4762. [21] R. de L. Kronig, On the theory of dispersion of X-rays, Journal of the Optical Society of America, 12 (1926) 547-557. 
[22] H.A. Kramers, Die dispersion und absorption von Roentgenstrahlen, Physikalishce Zeitschrift, 30 (1929) 522-523.

[23] P. Agarwal, M.E. Orazem, L.H. Garcia-Rubio, Measurement models for electrochemical impedance spectroscopy, Journal of The Electrochemical Society, 139 (1992) 1917.

[24] B.A. Boukamp, Practical application of the Kramers-Kronig transformation on impedance measurements in solid state electrochemistry, Solid State Ionics, 62 (1993) 131-141.

[25] P. Agarwal, M.E. Orazem, L.H. Garcia-Rubio, Application of measurement models to impedance spectroscopy: III. evaluation of consistency with the Kramers-Kronig relations Journal of The Electrochemical Society, 142 (1995) 4159.

[26] J.J. Giner-Sanz, E.M. Ortega, V. Pérez-Herranz, Application of a Montecarlo based quantitative Kramers-Kronig test for linearity assessment of EIS measurements, Electrochimica Acta, 209 (2016) 254-268.

[27] M. Durbha, M.E. Orazem, L.H. Garcia-Rubio, Spectroscopy applications of the KramersKronig transforms: implications for error structure identification, Journal of The Electrochemical Society, 144 (1997) 48.

[28] M.S.R. Urquidi-Macdonald, D.D. Macdonald, Application of Kramers-Kronig transforms in the analysis of electrochemical impedance data, Journal of The Electrochemical Society, 133 (1986) 2018.

[29] B.A. Boukamp, J. Ross Macdonald, Alternatives to Kronig-Kramers transformation and testing, and estimation of distributions, Solid State Ionics, 74 (1994) 85-101.

[30] A. Lasia, Electrochemical impedance spectroscopy and its applications, Springer (2014).

[31] F.W. King, Hilbert transforms, Cambridge University Press, Cambridge, 2009.

[32] N.E. Huang, Z. Shen, S.R. Long, A new view of nonlinear water waves: the Hilbert Spectrum, Annual Review of Fluid Mechanics, 31 (1999) 417-457.

[33] P.O. Bowles, T.C. Corke, D.G. Coleman, F.O. Thomas, M. Wasikowski, Improved Understanding of Aerodynamic Damping Through the Hilbert Transform, AIAA Journal, 52 (2014) 2384-2394.

[34] P. Sulzer, K. Oguchi, J. Huster, M. Kizmann, T.L.M. Guedes, A. Liehl, C. Beckh, A.S. Moskalenko, G. Burkard, D.V. Seletskiy, A. Leitenstorfer, Determination of the electric field and its Hilbert transform in femtosecond electro-optic sampling, Physical Review A, 101 (2020) 033821. 
[35] G. Zhang, Y. Li, T. Wang, H. Du, F. Luo, Y. Zhan, Extended Hilbert transform and application for seismic attributes, Earth and Space Science, 6 (2019) 873-886.

[36] J.A.C. Weideman, Computing the Hilbert transform on the real line, Mathematics of Computation, 64 (1995) 745-762.

[37] S. Olver, Computing the Hilbert transform and its inverse, Mathematics of Computation, 80 (2011) 1745-1767.

[38] H. Boche, V. Pohl, Limits of calculating the finite Hilbert transform from discrete samples, Applied and Computational Harmonic Analysis, 46 (2019) 66-93.

[39] H. Boche, V. Pohl, Investigations on the approximability and computability of the Hilbert transform with applications, Applied and Computational Harmonic Analysis, 48 (2020) 706730.

[40] T.H. Wan, M. Saccoccio, C. Chen, F. Ciucci, Influence of the discretization methods on the distribution of relaxation times deconvolution: Implementing radial Basis functions with DRTtools, Electrochimica Acta, 184 (2015) 483-499.

[41] T. Ivers, Eacute, E. E, A. Weber, eacute, Evaluation of electrochemical impedance spectra by the distribution of relaxation times, Journal of the Ceramic Society of Japan, 125 (2017) 193-201.

[42] M. Saccoccio, T.H. Wan, C. Chen, F. Ciucci, Optimal regularization in distribution of relaxation times applied to electrochemical impedance spectroscopy: ridge and lasso regression methods - a theoretical and experimental study, Electrochimica Acta, 147 (2014) 470-482.

[43] F. Ciucci, C. Chen, Analysis of electrochemical impedance spectroscopy data using the distribution of relaxation times: A Bayesian and hierarchical Bayesian approach, Electrochimica Acta, 167 (2015) 439-454.

[44] M.B. Effat, F. Ciucci, Bayesian and hierarchical Bayesian based regularization for deconvolving the distribution of relaxation times from electrochemical impedance spectroscopy data, Electrochimica Acta, 247 (2017) 1117-1129.

[45] C.M. Bishop, Pattern recognition and machine learning (Information Science and Statistics), Springer-Verlag2006.

[46] D.J.C. MacKay, Hyperparameters: Optimize, or integrate out?, in: G.R. Heidbreder (Ed.) Maximum entropy and Bayesian methods: Santa Barbara, California, U.S.A., 1993, Springer Netherlands, Dordrecht, 1996, pp. 43-59. 
[47] L. Pardo, Statistical inference based on divergence measures, Chapman and Hall press, 2005.

[48] K.P. Murphy, Machine learning: a probabilistic perspective, MIT press2012.

[49] J. Liu, F. Ciucci, The Gaussian process distribution of relaxation times: A machine learning tool for the analysis and prediction of electrochemical impedance spectroscopy data, Electrochimica Acta, 331 (2020) 135316.

[50] J. Liu, F. Ciucci, The Deep-Prior distribution of relaxation times, Journal of The Electrochemical Society, 167 (2020) 026506.

[51] J. Liu, J. Wang, A. Belotti, F. Ciucci, P-substituted $\mathrm{Ba}_{0.95} \mathrm{Laa}_{0.05} \mathrm{FeO}_{3-\delta}$ as a cathode material for SOFCs, ACS Applied Energy Materials, 2 (2019) 5472-5480. 
Table 1 - Exact EIS responses used for the stochastic experiments.

\begin{tabular}{|c|c|c|c|}
\hline Model & $Z(f)$ & Reference & $\begin{array}{l}\text { Ref. } \\
\text { Figures }\end{array}$ \\
\hline ZARC & $R_{\infty}+\frac{R_{\mathrm{ct}}}{1+\left(i 2 \pi f \tau_{0}\right)^{\phi}}$ & [30] & $2-4$ \\
\hline $2 \times \mathrm{ZARC}$ & $R_{\infty}+\frac{R_{\mathrm{ct}, 1}}{1+\left(i 2 \pi f \tau_{1}\right)^{\phi_{1}}}+\frac{R_{\mathrm{ct}, 2}}{1+\left(i 2 \pi f \tau_{2}\right)^{\phi_{2}}}$ & [30] & $5-6$ \\
\hline $\begin{array}{l}\text { Piece-wise } \\
\text { Constant } \\
\text { (PWC) }\end{array}$ & $R_{\infty}+\frac{R_{\mathrm{ct}}}{\ln \frac{\tau_{2}}{\tau_{1}}}\left(\ln \left(1-\frac{i}{2 \pi f \tau_{1}}\right)-\ln \left(1-\frac{i}{2 \pi f \tau_{2}}\right)\right)$ & [43] & 7 \\
\hline $\mathrm{L}_{0}+\mathrm{ZARC}$ & $i \omega L_{0}+R_{\infty}+\frac{R_{\mathrm{ct}}}{1+\left(i 2 \pi f \tau_{0}\right)^{\phi}}$ & & $8-9$ \\
\hline $\begin{array}{c}\text { Failed } \\
\text { experiment }\end{array}$ & $R_{\infty}+\operatorname{Re}\left(\frac{R_{\mathrm{ct}}}{1+\left(i 2 \pi f \tau_{0}\right)^{\phi_{1}}}\right)+\operatorname{Im}\left(\frac{R_{\mathrm{ct}}}{1+\left(i 2 \pi f \tau_{0}\right)^{\phi_{2}}}\right)$ & & $10-11$ \\
\hline
\end{tabular}


Table 2 - Values of the circuit parameters utilized in the stochastic experiments.

\begin{tabular}{|l|l|l|l|l|l|l|}
\hline Model & $R_{\infty}(\Omega)$ & $R_{\mathrm{ct}}(\Omega)$ & $\tau_{0}(\mathrm{~s})$ & $\phi$ & $L_{0}(\mathrm{H})$ & $\begin{array}{l}\text { Ref. } \\
\text { Figures }\end{array}$ \\
\hline ZARC & 10 & 50 & 1.0 & 0.8 & & $2-4$ \\
\hline $2 \times$ ZARC & 20 & {$[50,50]$} & {$[0.1,10]$} & {$[0.8,0.8]$} & & $5 \& 6$ LHS \\
\hline $2 \times Z$ ZARC & 20 & {$[50,50]$} & {$[0.1,1.0]$} & {$[0.8,0.8]$} & & $5 \& 6$ RHS \\
\hline PWC & 10 & 50 & {$[10,0.1]$} & & & 7 \\
\hline L $_{0}+$ ZARC & 10 & 50 & 1.0 & 0.8 & $5.0 \times 10^{-4}$ & 8 \\
\hline $\mathrm{L}_{0}+$ ZARC & 10 & 50 & 1.0 & {$[0.8,1.0]$} & $5.0 \times 10^{-4}$ & $10-11$ \\
\hline
\end{tabular}


Table 3 - Scores obtained for synthetic and real experiments.

\begin{tabular}{|c|c|c|c|c|c|c|c|c|c|c|c|c|c|}
\hline \multirow[b]{2}{*}{ Element } & \multicolumn{6}{|c|}{ Residual } & \multicolumn{2}{|c|}{ Mean } & \multicolumn{2}{|c|}{$\begin{array}{l}\text { Hellinger } \\
\text { Distance }\end{array}$} & \multicolumn{2}{|c|}{$\begin{array}{c}\text { Jensen-Shannon } \\
\text { Discrepancy }\end{array}$} & \multirow{2}{*}{$\begin{array}{l}\text { Ref. } \\
\text { Figures }\end{array}$} \\
\hline & $s_{1 \sigma, \mathrm{re}}$ & $s_{2 \sigma, \mathrm{re}}$ & $s_{3 \sigma, \mathrm{re}}$ & $s_{1 \sigma, \mathrm{im}}$ & $S_{2 \sigma, \mathrm{im}}$ & $s_{3 \sigma, \mathrm{im}}$ & $s_{\mu, \mathrm{re}}$ & $s_{\mu, \mathrm{im}}$ & $s_{\mathrm{HD}, \mathrm{re}}$ & $s_{\mathrm{HD}, \mathrm{im}}$ & $s_{\text {JSD,re }}$ & $s_{\mathrm{JSD}, \mathrm{im}}$ & \\
\hline ZARC & 0.877 & 1.000 & 1.000 & 0.605 & 0.914 & 1.000 & 0.991 & 0.976 & 0.571 & 0.636 & 0.768 & 0.796 & $2-4$ \\
\hline $2 \times \mathrm{ZARC}$ & 0.741 & 1.000 & 1.000 & 0.667 & 0.951 & 1.000 & 0.995 & 0.972 & 0.626 & 0.606 & 0.793 & 0.744 & $5 \& 6$ LHS \\
\hline $2 \times \mathrm{ZARC}$ & 0.765 & 1.000 & 1.000 & 0.617 & 0.938 & 1.000 & 0.996 & 0.976 & 0.619 & 0.573 & 0.788 & 0.711 & $5 \& 6$ RHS \\
\hline PWC & 0.716 & 1.000 & 1.000 & 0.642 & 0.938 & 1.000 & 0.991 & 0.962 & 0.591 & 0.562 & 0.771 & 0.705 & 7 \\
\hline $\mathrm{L}_{0}+\mathrm{ZARC}$ & 0.877 & 1.000 & 1.000 & 0.605 & 0.914 & 1.000 & 0.991 & 0.976 & 0.571 & 0.636 & 0.768 & 0.796 & 8 \\
\hline Failed exp & 0.543 & 0.778 & 0.864 & 0.395 & 0.753 & 0.852 & 0.959 & 0.873 & 0.351 & 0.316 & 0.480 & 0.422 & $10-11$ \\
\hline LIB & 0.941 & 0.980 & 1.000 & 0.471 & 0.804 & 1.000 & 0.993 & 0.952 & 0.810 & 0.362 & 0.931 & 0.460 & 12 \\
\hline SOFC & 1.000 & 1.000 & 1.000 & 0.840 & 0.988 & 1.000 & 0.997 & 0.989 & 0.674 & 0.522 & 0.865 & 0.673 & 13 \\
\hline
\end{tabular}




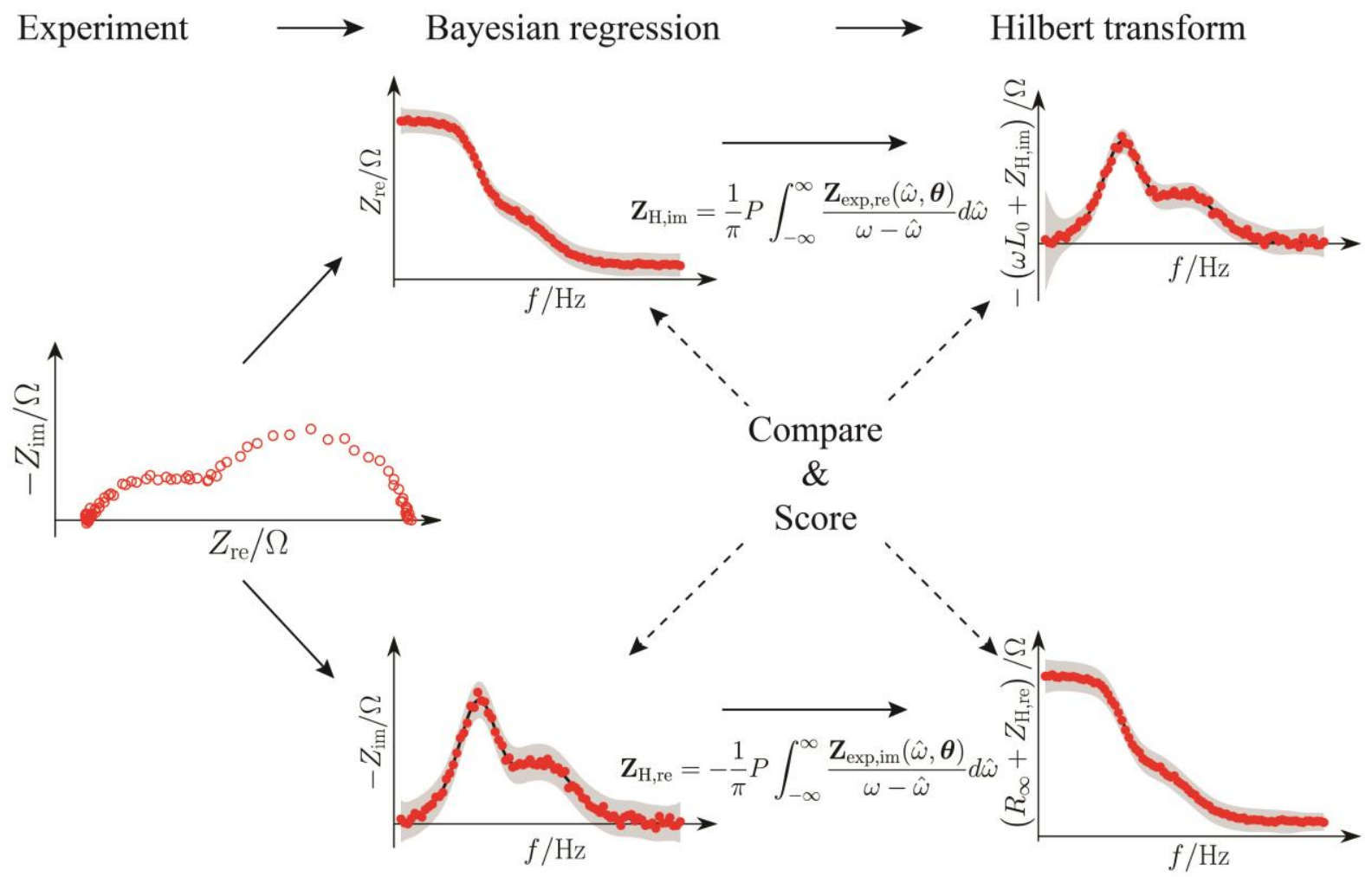

Figure 1 - Schematic illustration of the Bayesian Hilbert transform method as applied to electrochemical impedance spectroscopy data. 
(a)

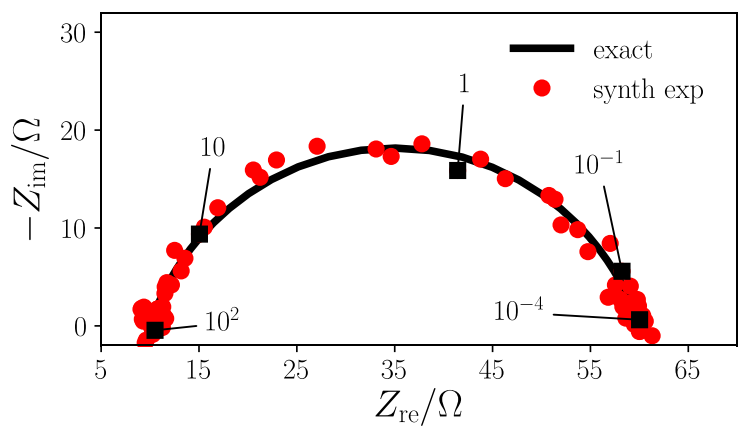

(b)

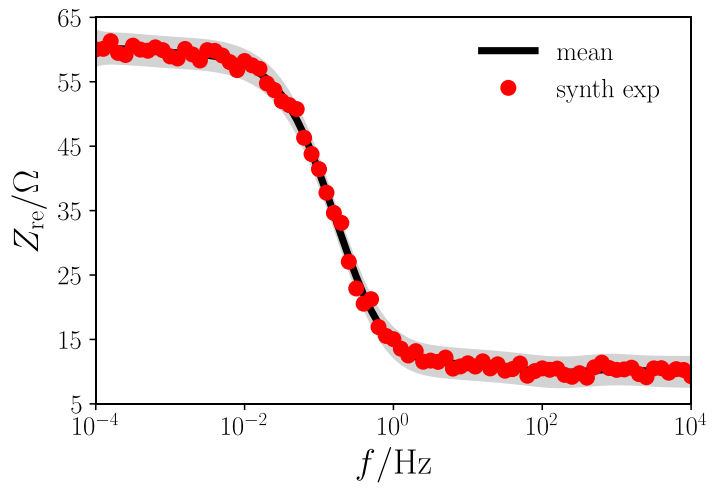

(c)

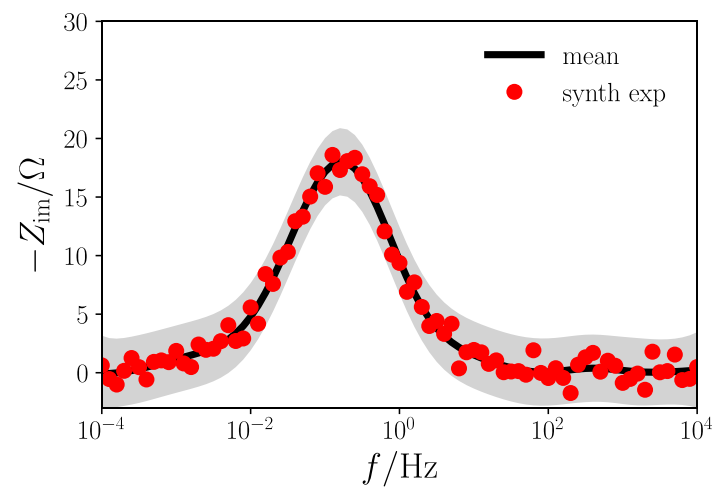

Figure 2 - (a) Nyquist plot of the synthetic experimental and exact impedance. (a) real and (b) imaginary parts of the impedance and Bayesian regression. Mean and $3 \sigma$ credible intervals are shown as a black line and a solid grey region, respectively. 
(a)

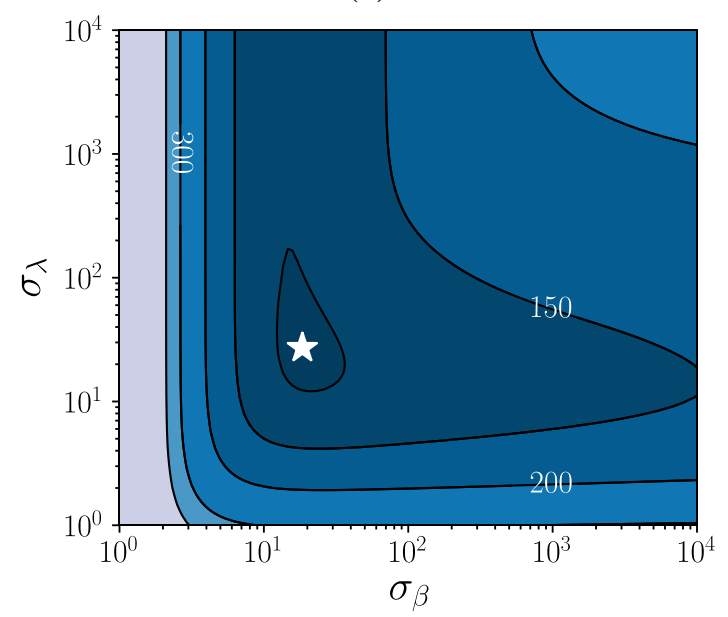

(b)

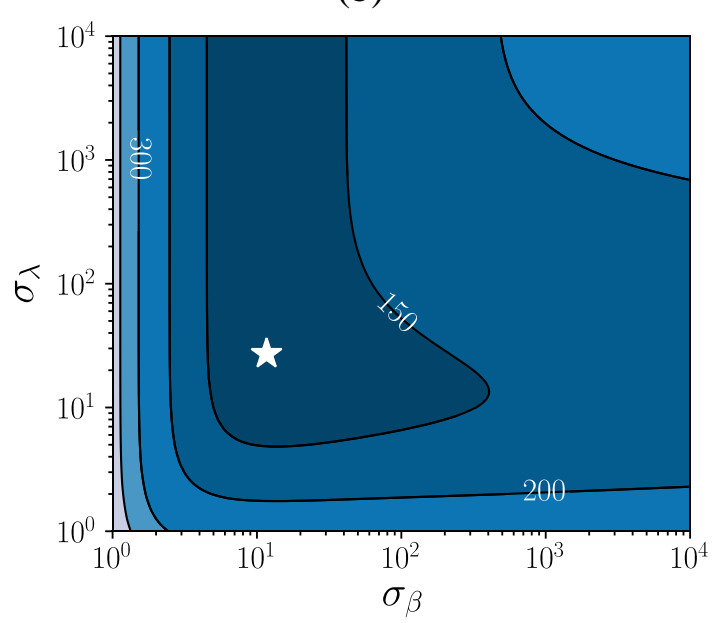

Figure 3 - Negative of the log-likelihood, $\mathcal{L}\left(\boldsymbol{\theta}, \boldsymbol{\omega}, \boldsymbol{Z}_{\text {exp }}\right)=-\log p\left(\boldsymbol{Z}_{\text {exp }} \mid \boldsymbol{\omega}, \boldsymbol{\theta}\right)$, computed for the (a) real and (b) imaginary of the impedance data of Figure 2. The $\sigma_{n}$ was set to be equal to $\sigma_{n, \exp }$ and the minimum is indicated with the star symbol. 
(a)

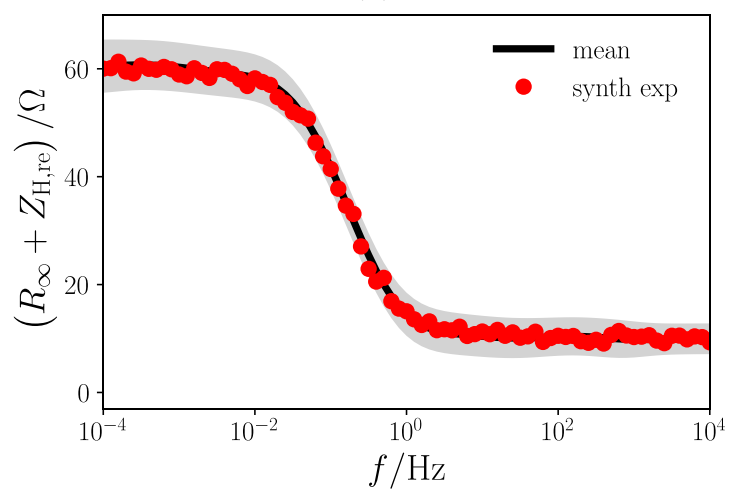

(c)

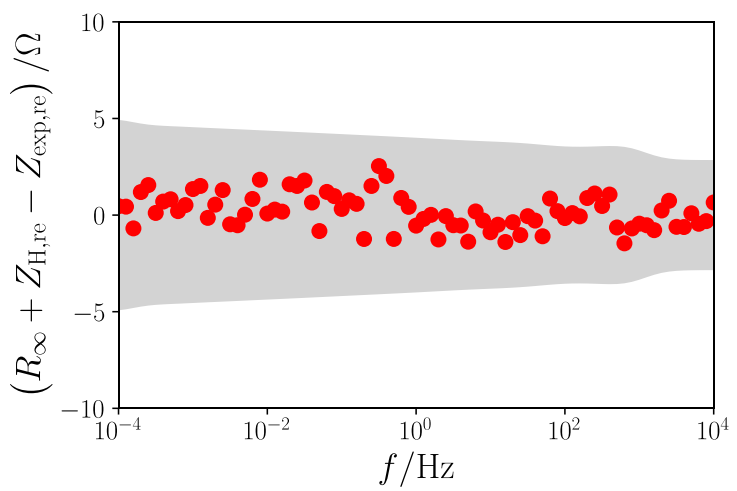

(e)

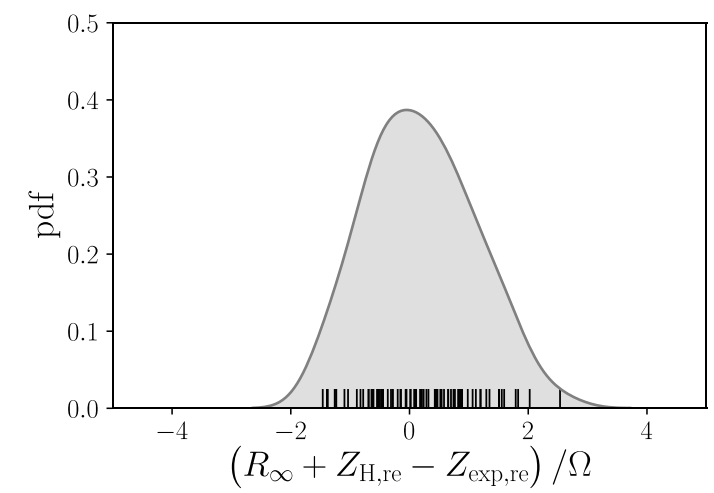

(b)

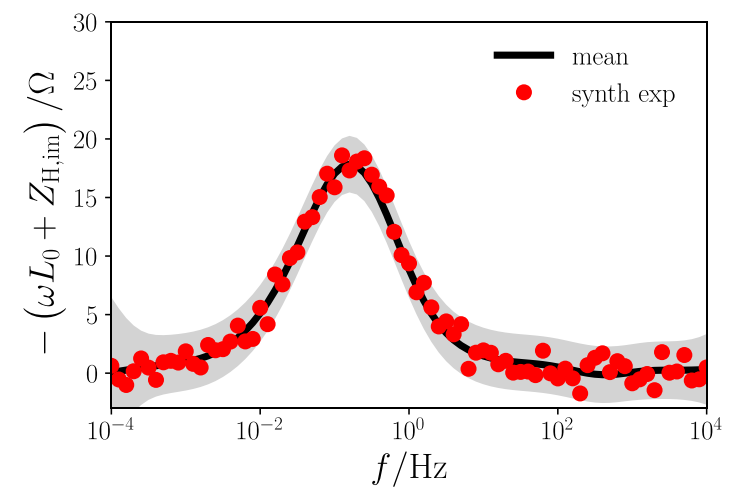

(d)

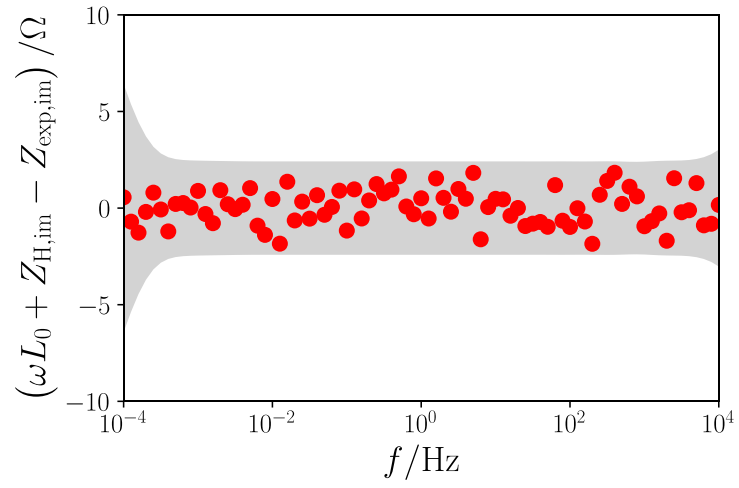

(f)

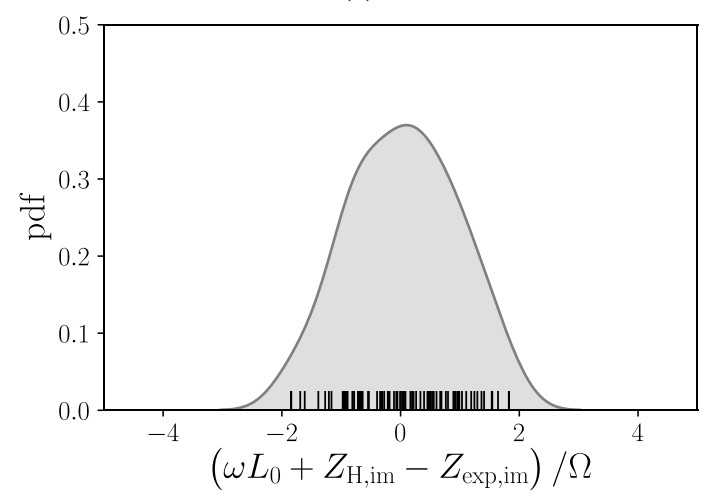

Figure 4 - (a) real and (b) imaginary part of the HT's data from Figure 2 with (c) \& (d) residuals and (e) \& (f) their distributions also shown. 
(a)

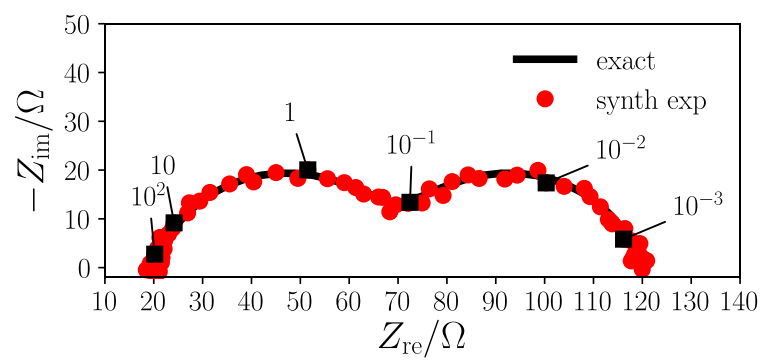

(c)

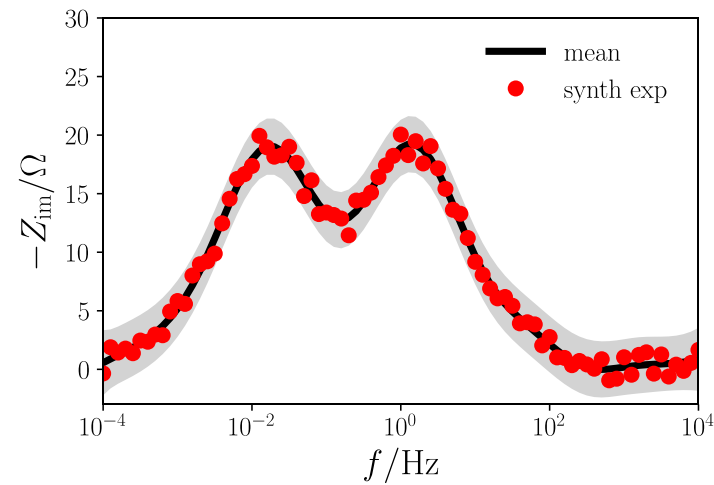

(b)

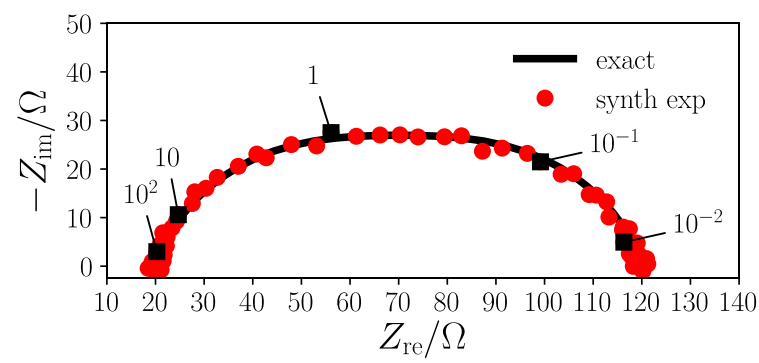

(d)

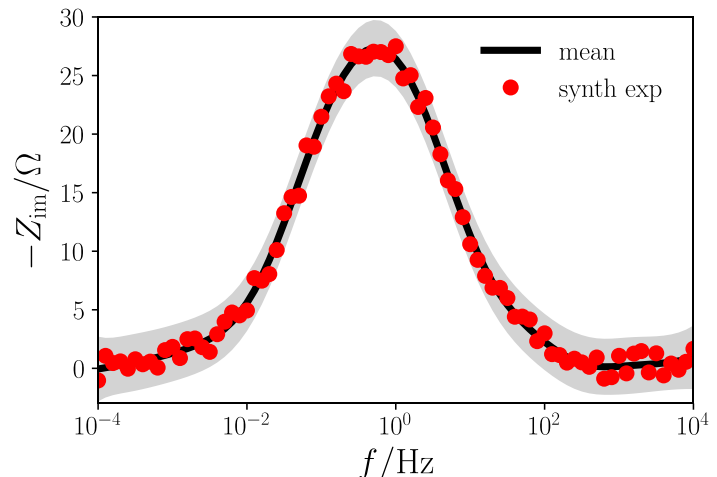

Figure 5 - Nyquist plot of the impedance response of two ZARC elements in series with (a) partially and (b) fully overlapping features. (c) and (d) imaginary part of the EIS spectrum as a function of the frequency and corresponding to the Nyquist plots in panels (a) and (b), respectively. 
(a)

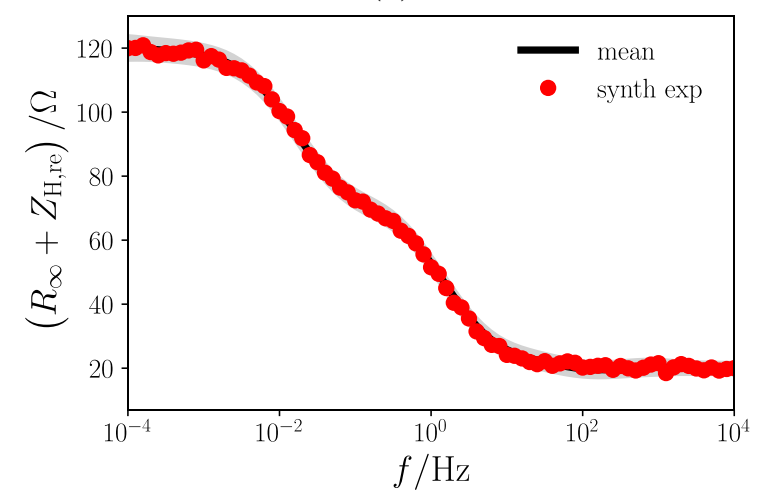

(c)

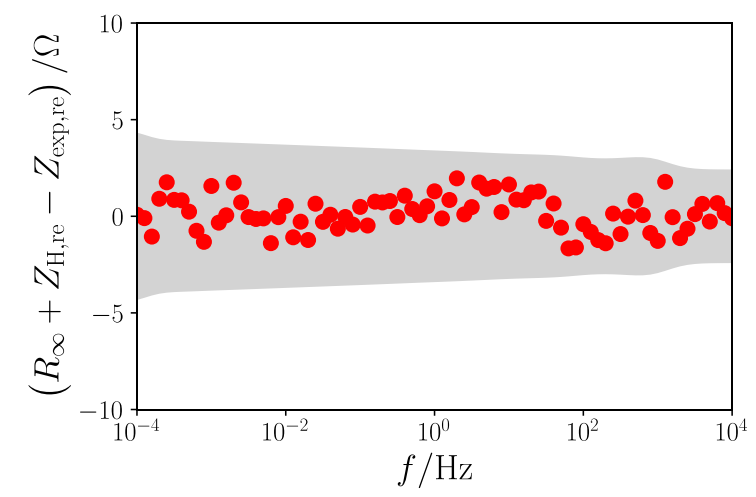

(e)

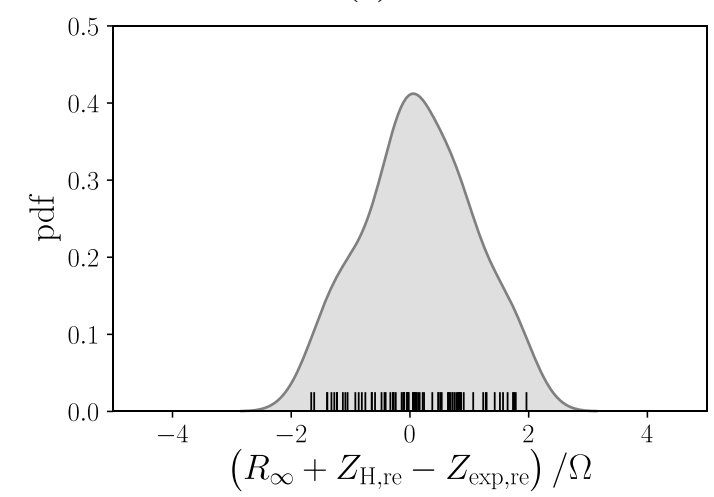

(b)

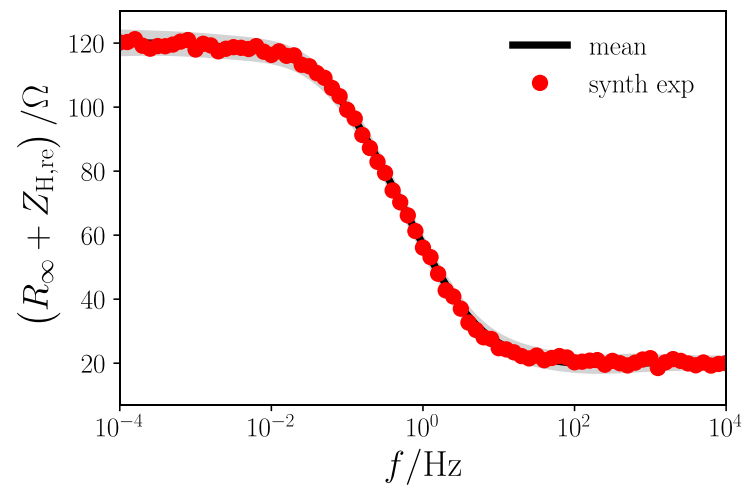

(d)

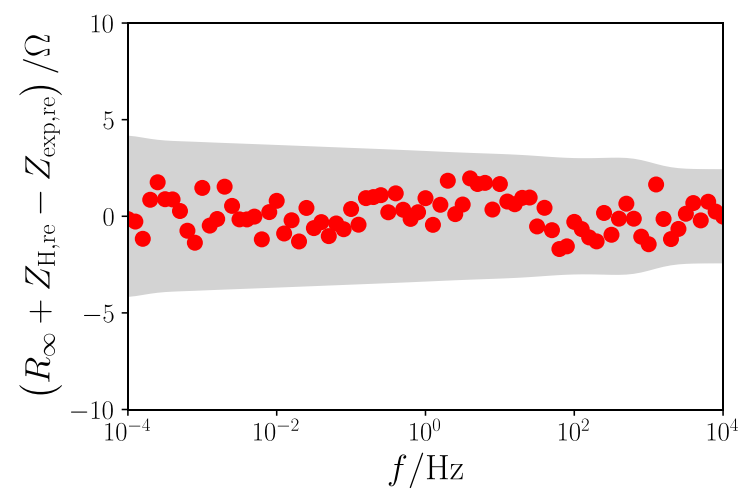

(f)

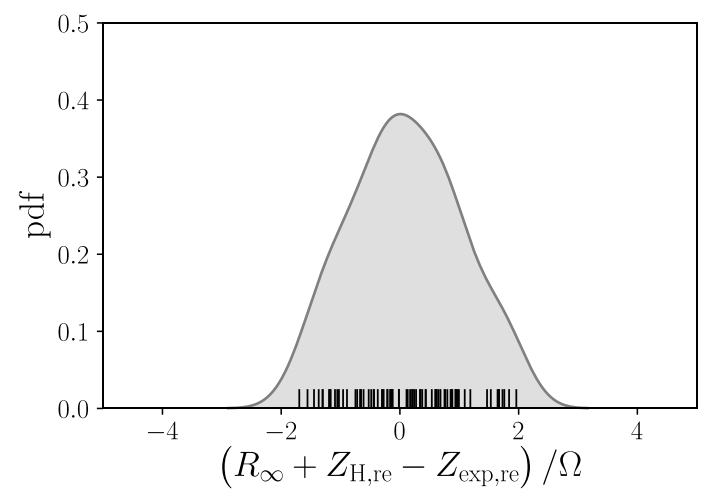

Figure 6 - (a) and (b) imaginary part of the HT's data with (c) \& (d) residuals and (e) \& (f) their distributions also shown. The left-hand and right-hand sides in this Figure correspond to the left-hand and right-hand sides of Figure 5. 
(a)

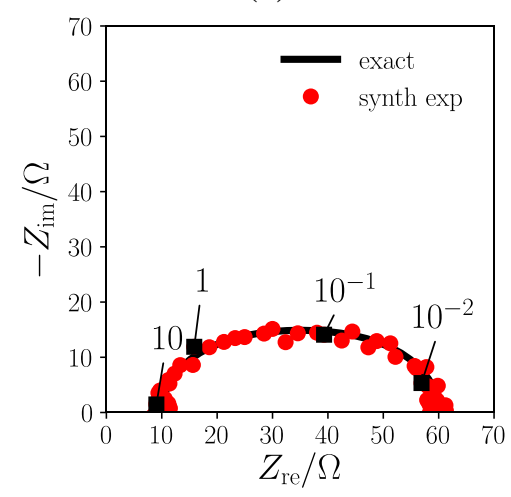

(c)

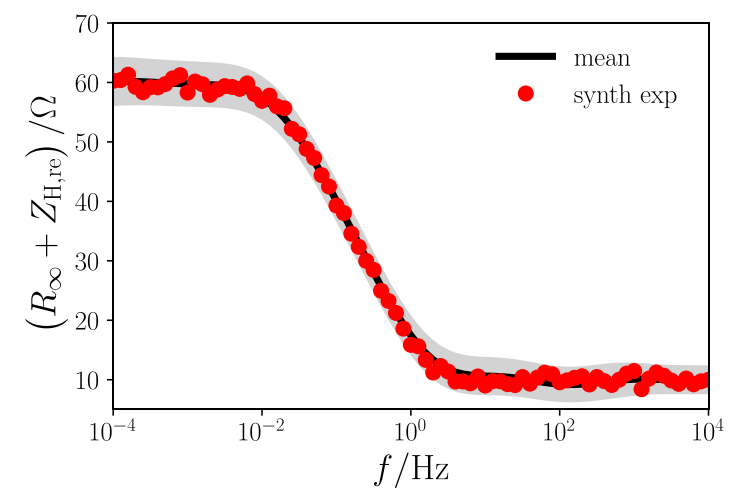

(b)

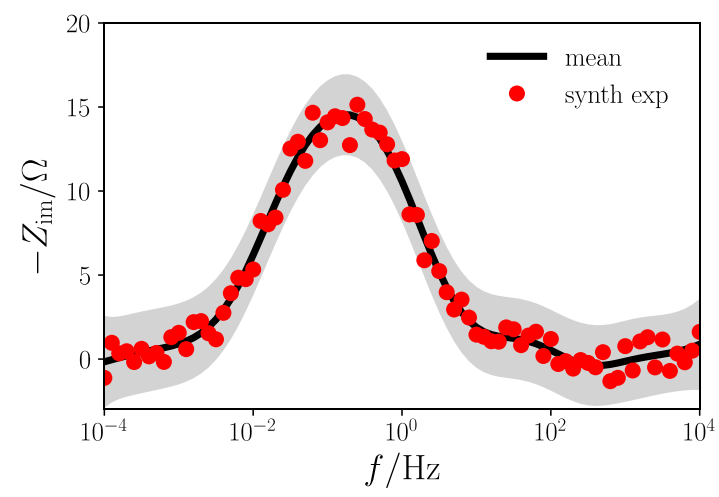

(d)

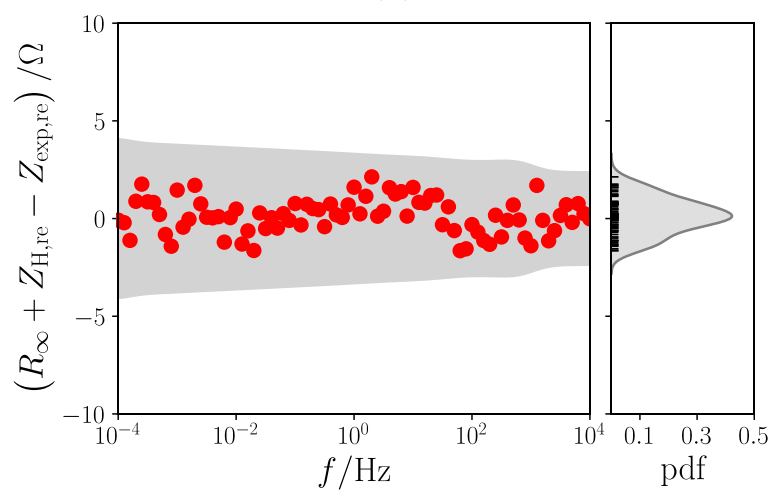

Figure 7 - (a) Nyquist plot of the EIS response of a PWC element. (b) The imaginary part of the EIS spectrum with Bayesian regression. (c) BHT of the $Z_{\text {exp,im }}$ and (d) corresponding residuals vs. frequency. 
(a)

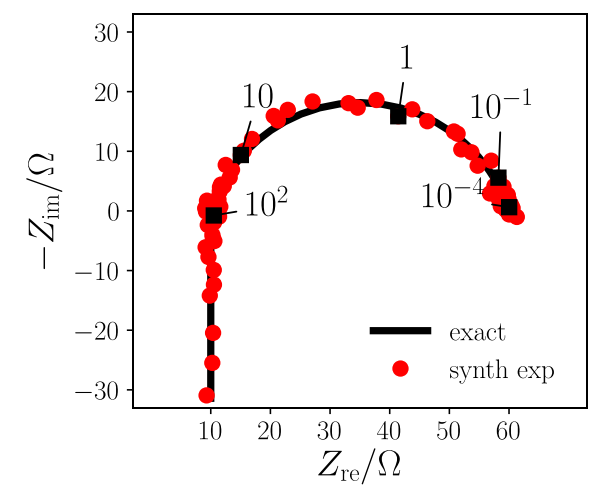

(c)

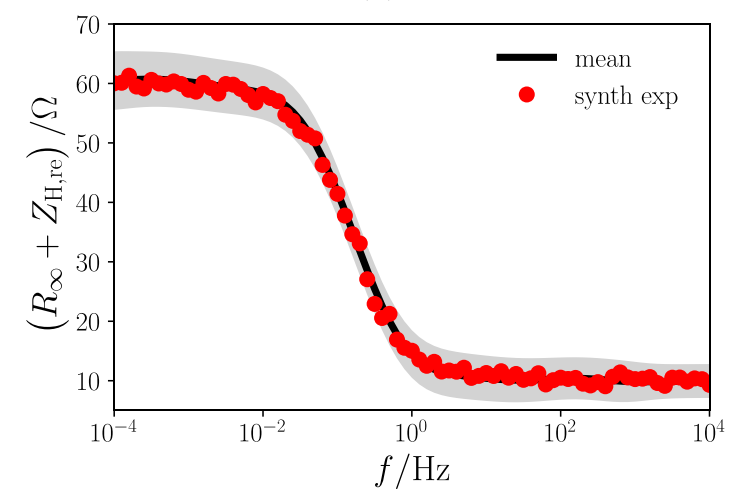

(b)

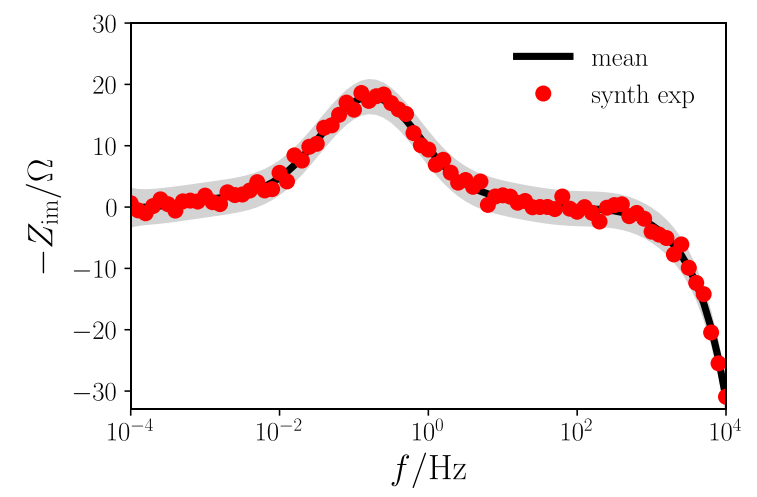

(d)

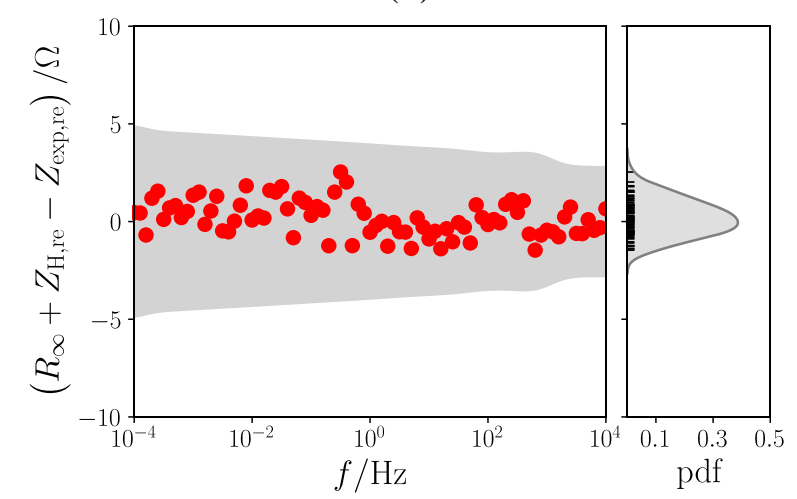

Figure 8 - (a) Nyquist plot of the EIS response of a ZARC element in series with a resistor and an inductor and (b) its regressed imaginary. (c) BHT of the $Z_{\text {exp,im }}$ and (d) corresponding residuals vs. frequency. 
(a)

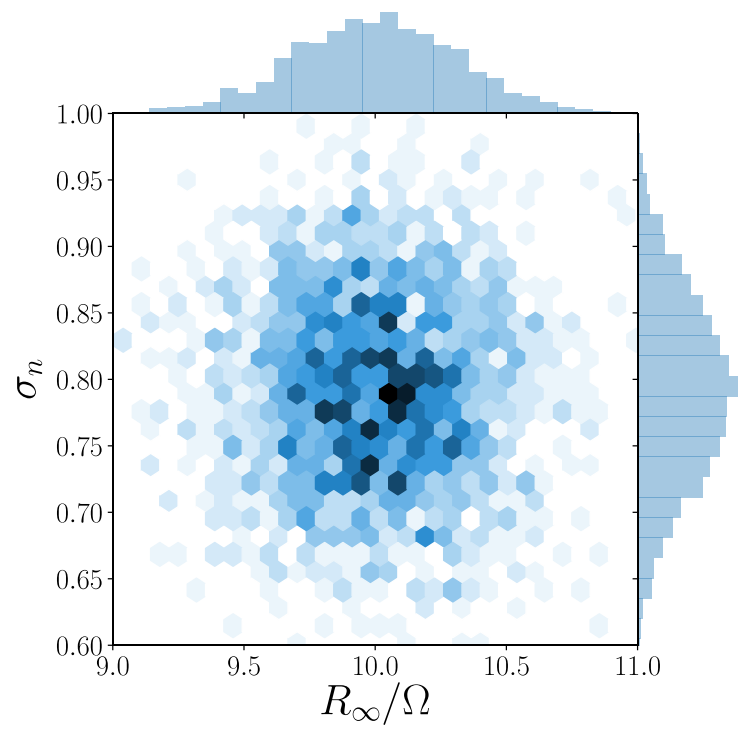

(b)

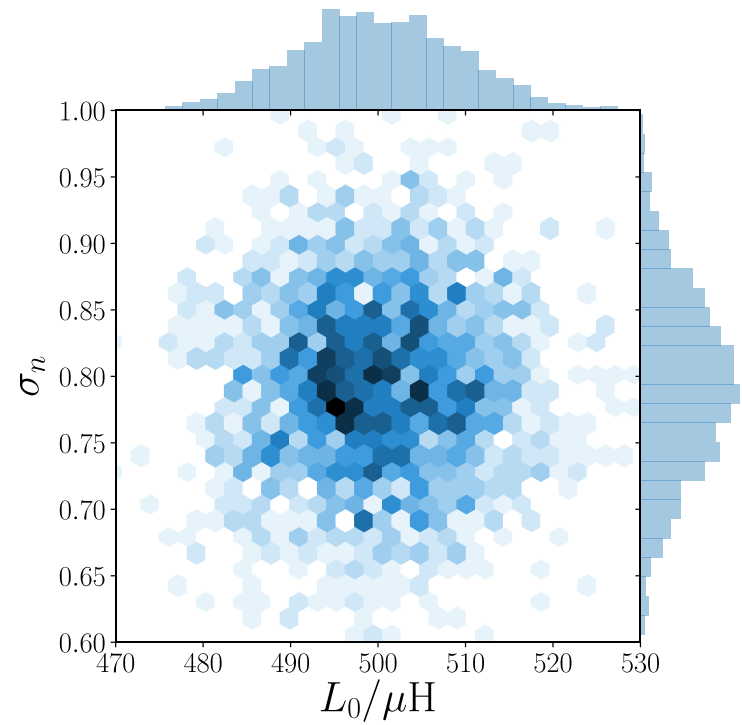

Figure 9 - Distribution of the optimal $\sigma_{n}$ as obtained by evidence maximization and regressed $R_{\infty}$ and $L_{0}$, the (a) imaginary and (b) real parts of the EIS spectrum were used to obtain these plots. 
(a)

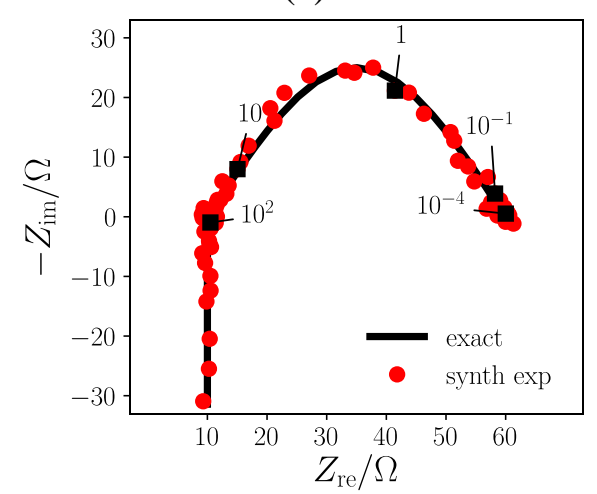

(b)

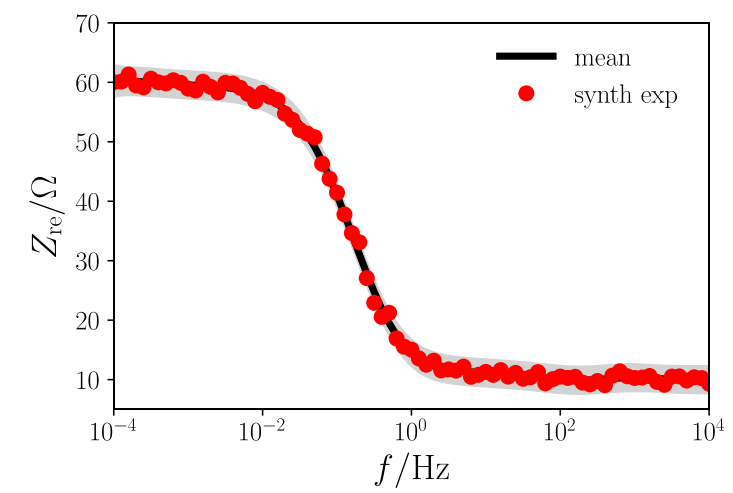

(c)

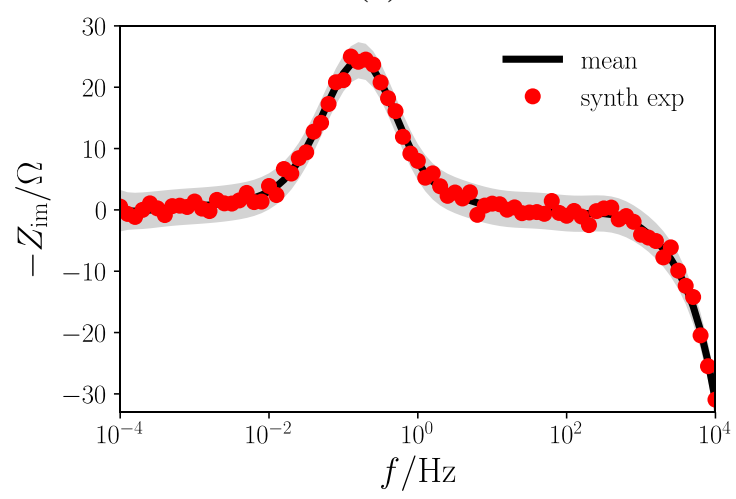

Figure 10 - (a) Nyquist plot of the synthetic experimental and exact impedance. (b) real and (c) imaginary parts of the impedance and Bayesian regression. Mean and $3 \sigma$ credible intervals are shown as a black line and a solid grey region, respectively. 
(a)

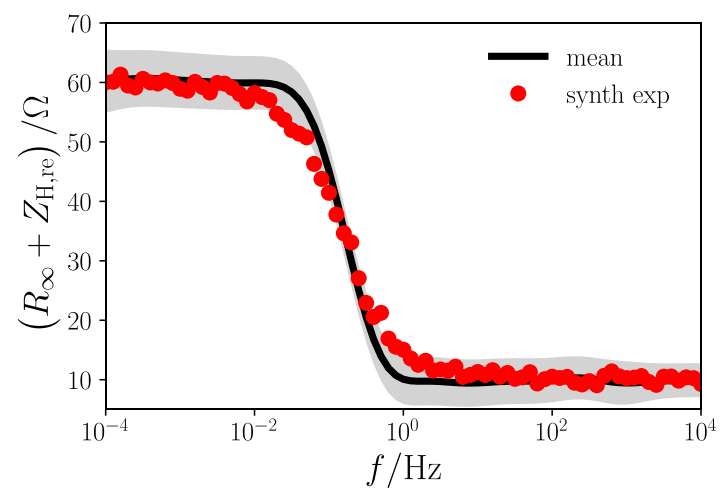

(c)

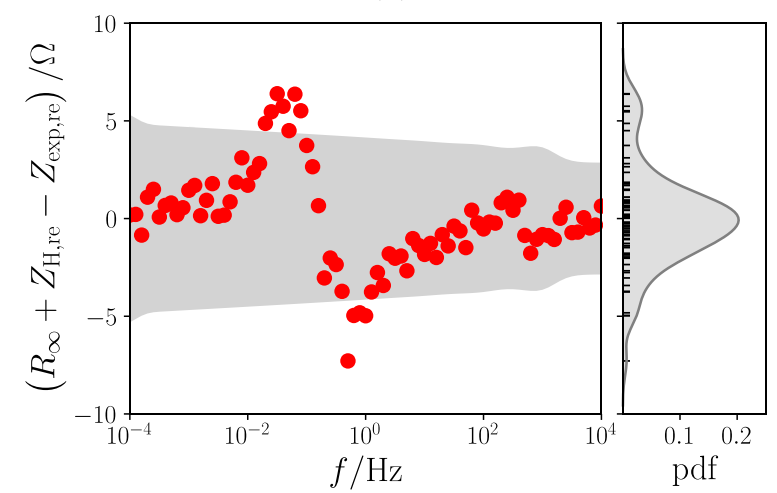

(b)

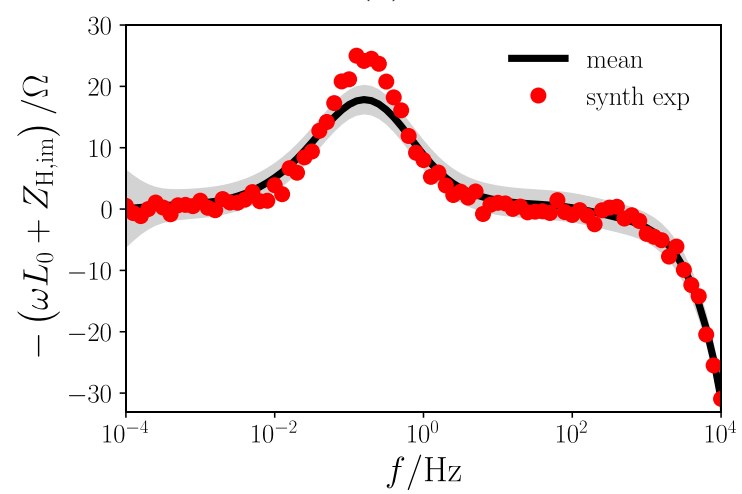

(d)

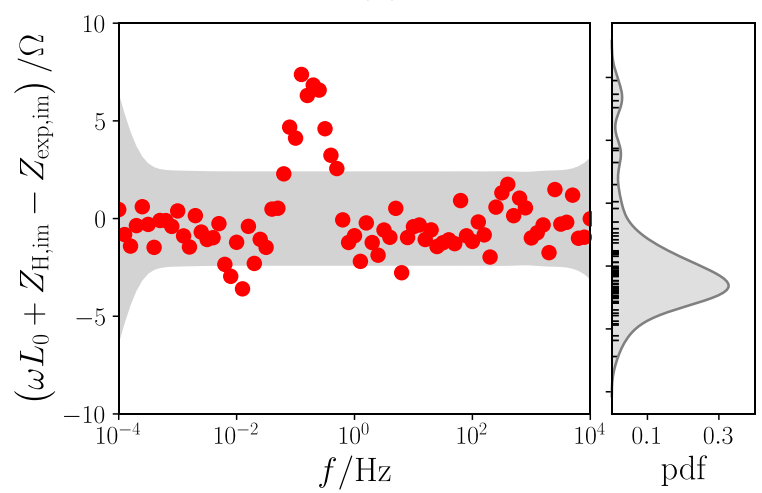

Figure 11 - (a) real and (b) imaginary part of the HT's data from Figure 10 with their (c) \& (d) residuals and distributions also shown. 
(a)

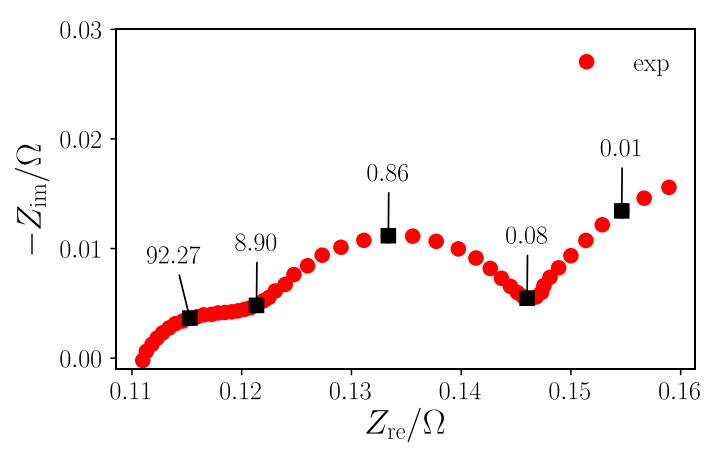

(c)

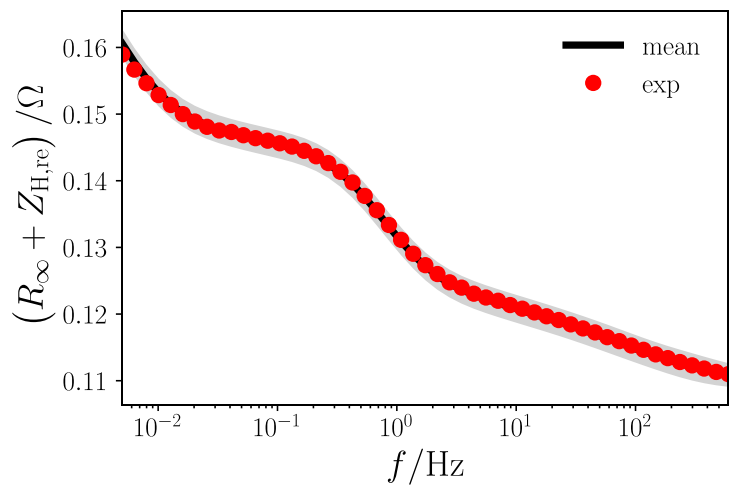

(b)

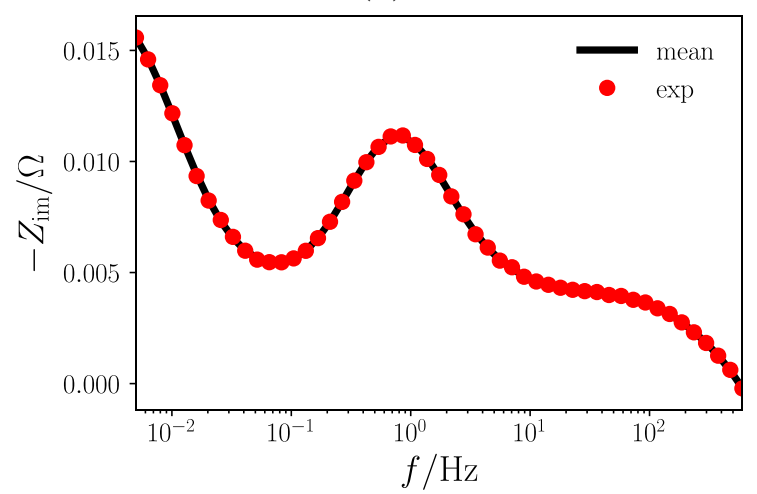

(d)

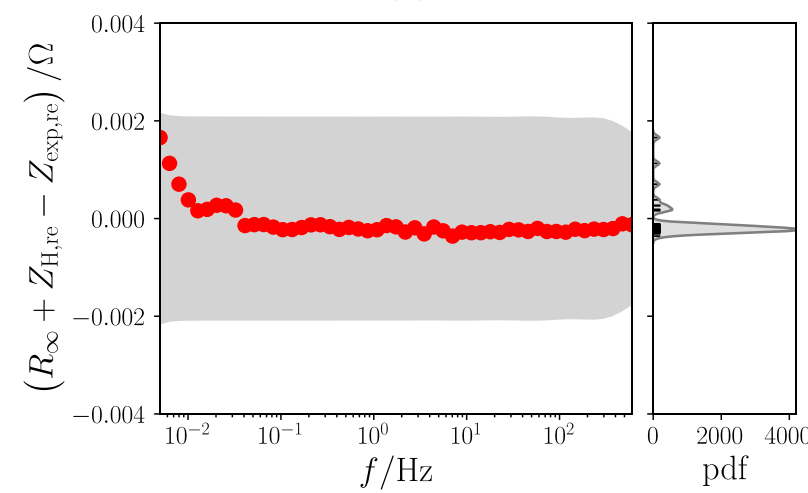

Figure 12 - (a) Nyquist plot of the EIS response of battery and (b) its regressed imaginary part.

(c) BHT of $Z_{\text {exp,im }}$ and (d) corresponding residuals vs. frequency with their distribution. 
(a)

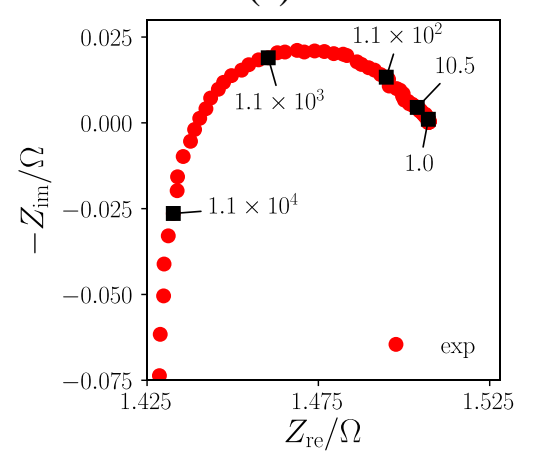

(c)

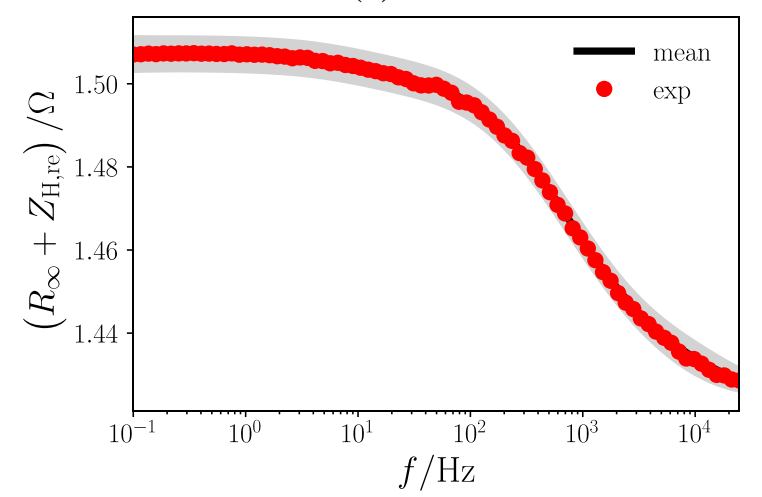

(b)

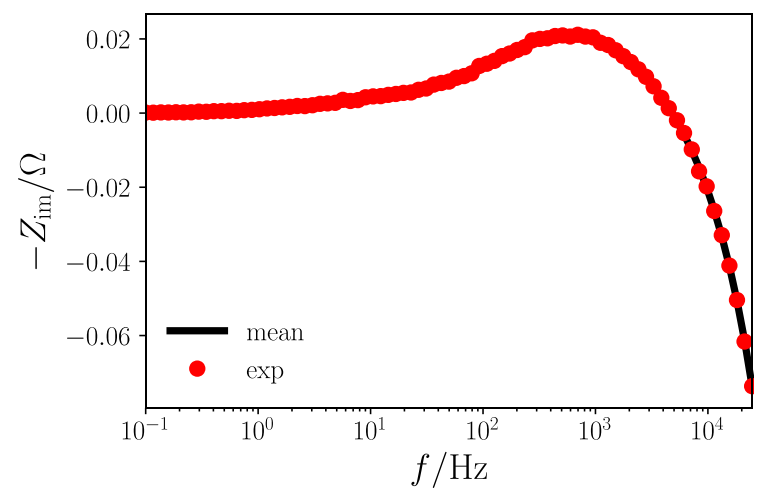

(d)

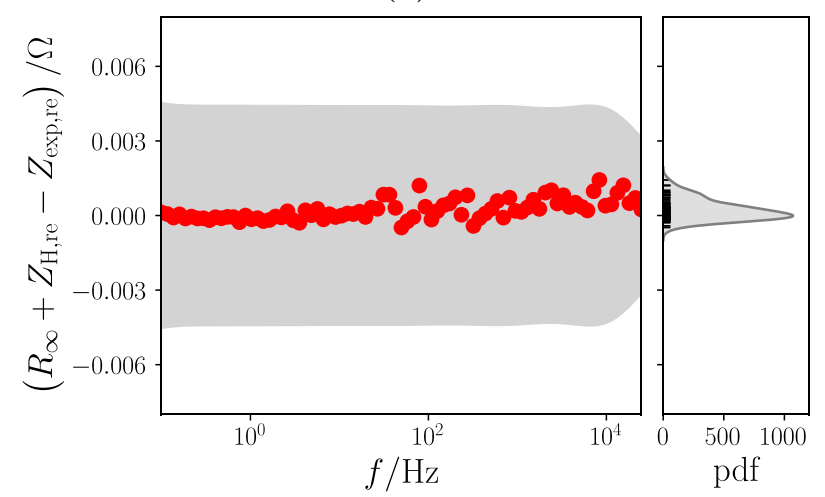

Figure 13 - (a) Nyquist plot of the BLFP|SDC|BLFP SOFC and (b) its regressed imaginary part.

(c) BHT of $Z_{\text {exp,im }}$ and (d) corresponding residuals vs. frequency with their distribution. 


\section{Appendix A Derivation of the Marginal Likelihood}

We start the derivation by recalling that the error model, (16), can be written as

$$
p\left(\boldsymbol{Z}_{\exp } \mid \boldsymbol{\omega}, \boldsymbol{x}, \sigma_{n}^{2}\right)=\frac{1}{\left(2 \pi \sigma_{n}^{2}\right)^{\frac{M}{2}}} \exp \left(-\frac{1}{2 \sigma_{n}^{2}}\left\|\boldsymbol{A} \boldsymbol{x}-\boldsymbol{Z}_{\exp }\right\|^{2}\right)
$$

and the prior, (17), is

$$
p\left(\boldsymbol{x} \mid \sigma_{\beta}^{2}, \sigma_{\lambda}^{2}\right)=\frac{|\boldsymbol{W}|^{\frac{1}{2}}}{(2 \pi)^{\frac{N+1}{2}}} \exp \left(-\frac{1}{2} \boldsymbol{x}^{\top} \boldsymbol{W} \boldsymbol{x}\right)
$$

where $\boldsymbol{W}$ depends on $\sigma_{\beta}^{2}$ and $\sigma_{\lambda}^{2}$. Therefore, the marginal likelihood can be obtained by integrating with respect to $x$ the product of (46) and (47):

$$
\begin{aligned}
p\left(\boldsymbol{Z}_{\exp } \mid \boldsymbol{\omega}, \boldsymbol{\theta}_{B}\right) & =\int_{\mathbb{R}^{N+1}} p\left(\boldsymbol{Z}_{\exp } \mid \boldsymbol{x}, \sigma_{n}^{2}\right) p\left(\boldsymbol{x} \mid \sigma_{\beta}^{2}, \sigma_{\lambda}^{2}\right) d \boldsymbol{x} \\
& =\frac{1}{\left(2 \pi \sigma_{n}^{2}\right)^{\frac{M}{2}}} \frac{|\boldsymbol{W}|^{\frac{1}{2}}}{(2 \pi)^{\frac{N+1}{2}}} \int_{\mathbb{R}^{N+1}} \exp (-E(\boldsymbol{x})) d \boldsymbol{x}
\end{aligned}
$$

where

$$
E(\boldsymbol{x})=\frac{1}{2 \sigma_{n}^{2}}\left\|\boldsymbol{A} \boldsymbol{x}-\boldsymbol{Z}_{\mathrm{exp}}\right\|^{2}+\frac{1}{2} \boldsymbol{x}^{\top} \boldsymbol{W} \boldsymbol{x}
$$

In order to integrate $\int_{\mathbb{R}^{N+1}} \exp (-E(\boldsymbol{x})) d \boldsymbol{x}$, we can "complete the square". This is done by grouping all terms with $\boldsymbol{x}$ in (49) and rewriting $E(\boldsymbol{x})$ as

$$
E(\boldsymbol{x})=E\left(\boldsymbol{\mu}_{x}\right)+\frac{1}{2}\left(\boldsymbol{x}-\boldsymbol{\mu}_{x}\right)^{\top} \boldsymbol{\Sigma}_{x}^{-1}\left(\boldsymbol{x}-\boldsymbol{\mu}_{x}\right)
$$


with $E\left(\boldsymbol{\mu}_{x}\right)$ as

$$
E\left(\boldsymbol{\mu}_{x}\right)=\frac{1}{2 \sigma_{n}^{2}}\left\|\boldsymbol{A} \boldsymbol{\mu}_{x}-Z_{\text {exp }}\right\|^{2}+\frac{1}{2} \boldsymbol{\mu}_{x}^{\top} \boldsymbol{W} \boldsymbol{\mu}_{x}
$$

It follows that

$$
\begin{aligned}
\int_{\mathbb{R}^{N+1}} \exp (-E(\boldsymbol{x})) d \boldsymbol{x} & \\
= & \exp \left(-E\left(\boldsymbol{\mu}_{x}\right)\right) \int_{\mathbb{R}^{N+1}} \exp \left(-\frac{1}{2}\left(\boldsymbol{x}-\boldsymbol{\mu}_{x}\right)^{\top} \boldsymbol{\Sigma}_{x}^{-1}\left(\boldsymbol{x}-\boldsymbol{\mu}_{x}\right)\right) d \boldsymbol{x}
\end{aligned}
$$

where the last integral can be computed exactly as

$$
\int_{\mathbb{R}^{N+1}} \exp \left(-\frac{1}{2}\left(\boldsymbol{x}-\boldsymbol{\mu}_{x}\right)^{\top} \boldsymbol{\Sigma}_{x}^{-1}\left(\boldsymbol{x}-\boldsymbol{\mu}_{x}\right)\right) d \boldsymbol{x}=(2 \pi)^{\frac{N+1}{2}}\left|\boldsymbol{\Sigma}_{x}\right|^{\frac{1}{2}}
$$

Using (52) and (53) in (48) allows us to write that

$$
p\left(\boldsymbol{Z}_{\exp } \mid \boldsymbol{\theta}\right)=\frac{1}{\left(2 \pi \sigma_{n}^{2}\right)^{\frac{M}{2}}}|\boldsymbol{W}|^{\frac{1}{2}}\left|\boldsymbol{\Sigma}_{x}\right|^{\frac{1}{2}} \exp \left(-E\left(\boldsymbol{\mu}_{x}\right)\right)
$$

We take the log of (54) to obtain the log-likelihood as

$$
\log p\left(\boldsymbol{Z}_{\text {exp }} \mid \boldsymbol{\theta}\right)=\frac{1}{2} \log |\boldsymbol{W}|+\frac{1}{2} \log \left|\boldsymbol{\Sigma}_{x}\right|-\frac{M}{2} \log \left(\sigma_{n}^{2}\right)-E\left(\boldsymbol{\mu}_{x}\right)-\frac{M}{2} \log (2 \pi)
$$

